\title{
Eikonal evolution and gluon radiation
}

\author{
Alex Kovner \\ Theory Division, CERN, CH-1211 Geneva 23, Switzerland \\ and Department of Mathematics and Statistics, University of Plymouth, Plymouth PL4 8AA, United Kingdom \\ Urs Achim Wiedemann \\ Theory Division, CERN, CH-1211 Geneva 23, Switzerland \\ (Received 27 June 2001; published 19 October 2001)
}

\begin{abstract}
We give a simple quantum mechanical formulation of the eikonal propagation approximation, which has been heavily used in recent years in problems involving hadronic interactions at high energy. This provides a unified framework for several approaches existing in the literature. We illustrate this scheme by calculating the total, elastic, inelastic and diffractive DIS cross sections, as well as gluon production in high energy hadronic collisions. From the $q \bar{q} g$ component of the DIS cross sections, we straightforwardly derive low $x$ evolution equations for inelastic and diffractive DIS distribution functions. In all calculations, we provide all order $1 / N$ corrections to the results existing in the literature.
\end{abstract}

DOI: 10.1103/PhysRevD.64.114002

PACS number(s): $13.60 . \mathrm{Hb}$

\section{INTRODUCTION}

In recent years a lot of work has been done aimed at understanding the physics of hadronic interactions in a dense (nuclear) environment. The questions addressed in this context range from deep inelastic scattering (DIS) at low $x$ to high energy scattering on nuclear targets to heavy ion collisions. Experimental motivations come from the low $x$ data measured at the DESY collider and the Fermilab Tevatron and, of course, heavy ion collision experiments at the BNL Relativistic Heavy Ion Collider (RHIC) and CERN Large Hadron Collider (LHC). An additional theoretical motivation is that the dense partonic environment provides a link between straight perturbative QCD of factorization theorems and Dokshitzer-Gribov-Lipatov-Altarelli-Parisi (DGLAP) evolution, and the still poorly understood soft QCD physics. The main idea is that high partonic density might provide an effective infrared cutoff, so that the genuine infrared strongly interacting region becomes relatively unimportant. The contribution of the infrared region to various observables which are not infrared safe in the usual perturbative sense, then may be suppressed by powers of $\Lambda / \mu$, where $\Lambda$ is the infrared scale and $\mu$ is the scale roughly measuring the strength of the gluon fields in the dense partonic system, $\mu \propto g A$. If $\mu$ is large enough, the coupling constant governing important interactions is small, and many non-infrared safe quantities may become in principle calculable. Although we are still far from the quantitative understanding of this interesting regime, progress is being continuously made in different approaches [1-15].

In this paper our aim is to give a simple physical description and provide a transparent calculational framework to what one may call eikonal propagation approach. The eikonal picture views a high energy hadronic reaction as a projectile, consisting of a (usually small) number of partons impinging on a large target. The target could be either a nucleus in its rest frame or a high energy hadron which has already developed many partons in its wave function. The interaction between the projectile partons and the target fields is assumed to be eikonal: that is the projectile partons propagate through the target without changing their transverse position but picking up an eikonal phase. As a result of the phase differences accumulated by the partons, the initial wave function decoheres and a different hadronic final state is produced. The target in this picture is described by an ensemble of gluon field configurations, and the averaging over this ensemble determines the cross section for various processes.

Eikonal physics underlies recent approaches towards calculating the produced particle multiplicities in high energy hadron-nucleus [5] and nucleus-nucleus [16] collisions. It is applied in the calculations of radiative jet energy loss in hot and cold nuclear matter [2] as well as in many studies of low $x$ DIS. A particularly lucid discussion with many explicit calculations is given by Buchmüller et al. [3]. Moreover, as discussed in Ref. [17], eikonal propagation is closely linked to the low $x$ evolution of Refs. $[7,8]$. On the other hand, the eikonal approach has been criticized in [11], since it does not take properly into account production of diffractive intermediate states, which should be equally important at high energy [18]. One of the main motivations for our work is indeed to set up a simple framework of eikonal propagation which can be extended to include diffractive production. While the present paper does not include diffractive production, we view it as a step towards this goal [19].

This paper is organized as follows: In Sec. II we give an explicit quantum mechanical description of the eikonal evolution and show how to calculate various cross sections from the knowledge of the outgoing wave function of the projectile that has undergone eikonal scattering. We explain how to perform averages over the gluon fields of the target. It turns out that the correct averaging procedure amounts to averaging on the amplitude level for the elastic and inelastic cross section, but on the cross section level for the diffractive one.

As a first illustration of our calculational scheme, we derive in Sec. III the gluon radiation in a hadron-nucleus scat- 
tering. This calculation reproduces the results of Ref. [5] for $q-A$ and $q \bar{q}-A$ systems.

In Sec. IV, we discuss deeply inelastic scattering on a large target. Our calculations are consistent with the results of Buchmüller et al. [3] and provide the $1 / N$ corrections to them. The most interesting quantity here is the diffractive cross section. We explain why physically the $1 / N$ corrections in this case are precisely what distinguishes between the elastic cross section calculated in $[3,20]$ and the diffractive, or large rapidity gap cross section that we calculate here. We calculate the diffractive cross section due to the $\bar{q} q$ as well as to the $\bar{q} q g$ component of the virtual photon.

In Sec. $\mathrm{V}$ we consider the low $x$ evolution in DIS. For the total photoabsorption cross section our results immediately yield the operator form of the low $x$ evolution equation of Ref. [7]. We derive $1 / N$ corrections to Kovchegov's version of this equation and show that these corrections express the fact that the fundamental dipoles do not propagate through the target independently beyond the leading order in $1 / N$. We also derive an analogous evolution equation for the diffractive cross section generalizing the results of Ref. [21]. Here we again obtain the operator form of the evolution, which is a new result. We show that in the large $N$ limit this equation reduces to that of Ref. [20] and we derive all-order $1 / N$ corrections to this equation within our model for the averaging over the target fields. We point out that both the leading and all-order $1 / N$ evolution is not an independent equation. Once the evolution of the total cross section is determined, the diffractive cross section is obtained by direct integration of the known function of rapidity.

Finally in Sec. VI we summarize our results.

\section{QUANTUM MECHANICS OF EIKONAL PROPAGATION}

Consider an energetic hadronic projectile impinging on a large nuclear target. The projectile is characterized by a wave function, in which the relevant degrees of freedom are the transverse positions and color states of the partons,

$$
\Psi_{\mathrm{in}}=\sum_{\left\{\alpha_{i}, x_{i}\right\}} \psi\left(\left\{\alpha_{i}, x_{i}\right\}\right)\left|\left\{\alpha_{i}, x_{i}\right\}\right\rangle
$$

The color index $\alpha_{i}$ can belong to the fundamental, antifundamental or adjoint representation of the color $S U(N)$ group, corresponding to quark, antiquark or gluon in the wave function. In what follows we will consider wave functions with a small number of partons.

\section{A. Projectile biased observables}

At high energy the propagation time through the target is short, and thus partons propagate independently of each other. For the same reason the transverse positions of the partons do not change during the propagation. The only effect of the propagation is that the wave function of each parton acquires an eikonal phase due to the interaction with the glue field of the target. Thus the projectile emerges from the interaction region with the wave function

$$
\Psi_{\text {out }}=\mathcal{S} \Psi_{\text {in }}=\sum_{\left\{\alpha_{i}, x_{i}\right\}} \psi\left(\left\{\alpha_{i}, x_{i}\right\}\right) \prod_{i} W\left(x_{i}\right)_{\alpha_{i} \beta_{i}}\left|\left\{\beta_{i}, x_{i}\right\}\right\rangle .
$$

Here $\mathcal{S}$ is the $S$ matrix, and the $W$ 's are Wilson lines:

$$
W\left(x_{i}\right)=\mathcal{P} \exp \left\{i \int d z_{-} T^{a} A_{a}^{+}\left(x_{i}, z_{-}\right)\right\}
$$

with $A^{+}$the gauge field in the target and $T^{a}$ the generator of $S U(N)$ in a representation corresponding to a given parton. Thus the relative phases between components of the wave function change, and the state that emerges after the target is no longer an eigenstate of the strong interaction Hamiltonian (as the incoming state is assumed to be) but rather a superposition thereof.

Given this explicit law for the evolution of the projectile wave function, one can calculate various observables related to the scattering process. For example, up to the factor of total flux, the inelastic cross section is proportional to the probability that the outgoing state is different from the incoming one. This probability is given by the norm

$$
P_{\mathrm{inel}}^{\mathrm{proj}}=|\delta \Psi|^{2},
$$

where

$$
|\delta \Psi\rangle=\left[1-\left|\Psi_{\text {in }}\right\rangle\left\langle\Psi_{\text {in }}\right|\right]\left|\Psi_{\text {out }}\right\rangle=\left|\Psi_{\text {out }}\right\rangle-s\left|\Psi_{\text {in }}\right\rangle,
$$

and $s$ is the $S$-matrix element, or overlap

$$
s=\left\langle\Psi_{\text {in }} \mid \Psi_{\text {out }}\right\rangle .
$$

Thus

$$
P_{\text {inel }}^{\text {proj }}=1-\left|\left\langle\Psi_{\text {in }} \mid \Psi_{\text {out }}\right\rangle\right|^{2} .
$$

The probability $P_{\text {inel }}^{\text {proj }}$ for a given configuration of the target fields of course depends on the impact parameter, that is the position of the projectile in the transverse plain. To get the cross section one simply integrates the probability over the impact parameter

$$
\sigma_{\text {inel }}^{\text {proj }}=\int d^{2} b P_{\text {inel }}^{\text {proj }}(b) .
$$

Note that the probability $P_{\text {inel }}^{\text {proj }}$ as defined above does not include processes for which the projectile remains intact but the target is excited or breaks into fragments that stay close to the original rapidity. By its very definition, $P_{\text {inel }}^{\text {proj }}$ is the probability that the projectile scatters inelastically. We refer to it as a projectile biased observable and we denote it by a superscript "proj". The formulation which removes this bias will be given in Sec. II C.

More generally, the incoming state may have internal degrees of freedom which we collectively denote by $\gamma$. This can be spin or (perturbatively) global color. In that case the inelastic probability is the probability that the outcoming state is perpendicular to any one of the states $\Psi_{\text {in }}(\gamma)$. Technically this means that $\delta \Psi$ is defined by subtracting from $\Psi_{\text {out }}$ its projection onto a subspace spanned by $\gamma$ : 


$$
|\delta \Psi\rangle=\left[1-\sum_{\gamma}\left|\Psi_{\text {in }}(\gamma)\right\rangle\left\langle\Psi_{\text {in }}(\gamma)\right|\right]\left|\Psi_{\text {out }}\right\rangle
$$

For orthonormal states $\Psi_{i n}(\gamma)$ the operator

$$
p_{\gamma}=1-\sum_{\gamma}\left|\Psi_{\text {in }}(\gamma)\right\rangle\left\langle\Psi_{\text {in }}(\gamma)\right|
$$

is the projector on the subspace orthogonal to the possible initial states of the projectile hadron.

One can also calculate other, less inclusive observables. For example the number of gluons with momentum $k$ produced in the scattering event is given by

$$
N_{\text {prod }}(\mathbf{k})=\left\langle\delta \Psi\left|a^{\dagger}(k) a(k)\right| \delta \Psi\right\rangle .
$$

In principle, the calculation of any observable in an inelastic process has to allow for the evolution of the state after the interaction to the time when the measurement is performed, that is $t=\infty$. Thus the relevant observable should be calculated not in the state $|\delta \Psi\rangle$, but rather in $U(0, \infty)|\delta \Psi\rangle$, where $U(0, \infty)$ is the free evolution operator from the time zero (immediately after the interaction) to infinity. Being a unitary operator, it cancels against its conjugate in the calculation of the cross section, Eq. (2.4), but it affects some less inclusive observables, such as the number of produced gluons (2.11). In the quantities we calculate in this paper its contribution is always a perturbative correction of higher order than the accuracy to which we are working, and we do not consider this type of correction. As will become apparent from our calculations in the later sections, this does not mean that we neglect contributions which in the Feynman diagram language correspond to "emission after interaction". Those in the present framework arise from the "overlap" contributions, proportional to the overlap $s$ of Eqs. (2.5),(2.6).

In contrast to the inelastic cross section, the total cross section includes the probability for an elastic process, for which the outcoming state is the same as the incoming one up to a phase shift. In this case, one includes in the calculation all components of the outgoing wave function which differ from the incoming one,

$$
\left|\delta \Psi_{\text {tot }}\right\rangle=\left|\Psi_{\text {out }}\right\rangle-\left|\Psi_{\text {in }}\right\rangle,
$$

and

$$
\begin{aligned}
\sigma_{\text {tot }} & =\int d^{2} b\left\langle\Psi_{\text {out }}(b)-\Psi_{\text {in }}(b) \mid \Psi_{\text {out }}(b)-\Psi_{\text {in }}(b)\right\rangle \\
& =\int d^{2} b\left[2-\left\langle\Psi_{\text {out }}(b)\right\rangle\left|\Psi_{\text {in }}(b)\right\rangle-\left\langle\Psi_{\text {in }}(b)\right\rangle\left|\Psi_{\text {out }}(b)\right\rangle\right] .
\end{aligned}
$$

The elastic cross section measures the probability of a process in which the product of the collision is in the same state as the initial one but with a nontrivial phase shift. This is defined through

$$
\left|\delta \Psi_{\text {el }}\right\rangle=\left[\left\langle\Psi_{\text {in }} \mid \Psi_{\text {out }}\right\rangle-1\right]\left|\Psi_{\text {in }}\right\rangle
$$

and

$$
\begin{aligned}
\sigma_{\mathrm{el}}^{\mathrm{proj}} & =\int d^{2} b\left\langle\delta \Psi_{\mathrm{el}} \mid \delta \Psi_{\mathrm{el}}\right\rangle \\
& \left.=\int d^{2} b\left|1-\left\langle\Psi_{\mathrm{out}}(b)\right\rangle\right| \Psi_{\text {in }}(b)\right\rangle\left.\right|^{2} .
\end{aligned}
$$

Another interesting quantity is the diffractive cross section. This includes all elastic and inelastic processes for which the outgoing state is different from the incoming one and is in a color singlet:

$$
\left|\delta \Psi_{\text {diff }}\right\rangle=\mathcal{P}_{\text {singlet }}\left|\Psi_{\text {out }}\right\rangle-\left|\Psi_{\text {in }}\right\rangle
$$

and

$$
\begin{aligned}
\sigma_{\text {diff }}= & \int d^{2} b\left\langle\mathcal{P}_{\text {singlet }} \Psi_{\text {out }}(b)-\Psi_{\text {in }}(b)\right| \mathcal{P}_{\text {singlet }} \Psi_{\text {out }}(b) \\
& \left.-\Psi_{\text {in }}(b)\right\rangle .
\end{aligned}
$$

The operator $\mathcal{P}$ is the projection operator on the color singlet states.

\section{B. Target averages}

The only property of the gluon field in the target assumed in the preceding discussion is that it does not vary during the time of the interaction. This is justified since the time of the propagation of an energetic projectile through the target is shorter than the natural time scale on which the target fields vary. The target is, however, characterized by a wave function which has a spread in field space. The relative phases of different components of this wave function are not relevant in the eikonal approximation, but the probability distribution for different field strengths is relevant indeed. In other words, while each scattering event happens on a frozen field profile, in order to calculate the cross section one has to average over the field ensemble which represents the wave function of the target.

In this paper we adopt the same ensemble averaging procedure as in Refs. $[2,22,5]$. The target is pictured as an assembly of sources of the color field extended in both the longitudinal and transverse directions. The sources are independent of each other and thus completely decorrelated. The longitudinal and transverse coordinates of the sources are randomly distributed within the target's longitudinal and transverse extent. The field emitted by each source is small, and to leading order in the field strength, the projectile can exchange only one or two gluons with each one of the sources. Pictorially, the average of, say a product of two Wilson loops can be represented as in Fig. 1.

Put into formulas, this means that the target average of the two-point function of the vector potential $A^{+}$, which enters all our calculations through Eqs. (2.2), (2.3) is

$$
\left\langle\left\langle A^{+a}\left(\mathbf{x}, x^{-}\right) A^{+b}\left(\mathbf{y}, y^{-}\right)\right\rangle\right\rangle=\delta^{a b} \delta\left(x^{-}-y^{-}\right) B\left(\mathbf{x}, \mathbf{y}, x^{-}\right) .
$$

All final results will depend on one particular linear combination of these averages: 


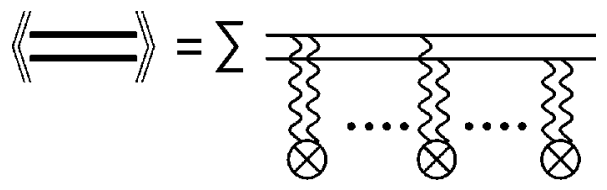

FIG. 1. The target average of the product of two Wilson lines, denoted by thick lines. The fields $A^{+}$in $W$ 's are distributed with Gaussian weights local in the longitudinal coordinate according to Eq. (2.18).

$$
v(\mathbf{x}, \mathbf{y})=\int d x^{-} \frac{1}{2}\left[B\left(\mathbf{x}, \mathbf{x}, x^{-}\right)+B\left(\mathbf{y}, \mathbf{y}, x^{-}\right)-2 B\left(\mathbf{x}, \mathbf{y}, x^{-}\right)\right] .
$$

For simplicity of notation we will sometimes consider the field ensemble to be translationally invariant in the transverse plain within some radius $R$, and will take all fields to vanish outside this radius. In this approximation, $v$ depends only on the difference of its coordinates.

With this averaging procedure, the target averages can be explicitly calculated. This is done for a variety of observables in Appendix C. The simplest averages we will need are those corresponding to "fundamental dipole" and "adjoint dipole" scattering amplitudes

$$
\frac{1}{N}\left\langle\left\langle\operatorname{Tr}\left[W^{F \dagger}(\mathbf{x}) W^{F}(\mathbf{y})\right]\right\rangle\right\rangle=\exp \left[-C_{F} v(\mathbf{x}-\mathbf{y})\right],
$$

$$
\frac{1}{N^{2}-1}\left\langle\left\langle\operatorname{Tr}\left[W^{A \dagger}(\mathbf{y}) W^{A}(\mathbf{x})\right]\right\rangle\right\rangle=\exp \left[-C_{A} v(\mathbf{x}-\mathbf{y})\right],
$$

with the Wilson loops in the fundamental and adjoint representations respectively.

In most of the paper we will not specify the form of $B$, but sometimes it is useful to keep in mind the following simple form $[5,22]$ :

$$
B(\mathbf{x}, \mathbf{y})=\mathbf{x} \cdot \mathbf{y} \mu^{2}\left(x^{-}\right),
$$

where $\mu$ is a slowly varying function of $x^{-}$. The independence of $\mu$ on $\mathbf{x}$ inside the radius of the target corresponds to the leading logarithmic approximation in the following sense. We are working in the gauge where the only nonvanishing component of the vector potential in the target is $A^{+}$. In this gauge the vector potential is related to the color electric field by the following relation which is local in $x^{-}$

$$
A^{+}(\mathbf{x})=\int_{\mathbf{x}_{\mathbf{0}}}^{\mathbf{x}} d x_{i} E_{i}
$$

The leading logarithmic approximation assumes that the scale of the variation of the color electric field is much larger than the transverse distances at which the field is probed (transverse momentum ordering). In this approximation, we have

$$
A^{+}(\mathbf{x})=\left(\mathbf{x}-\mathbf{x}_{\mathbf{0}}\right) \cdot \mathbf{E}
$$

and thus choosing $\mathbf{x}_{\mathbf{0}}=0$, Eq. (2.18) with Eq. (2.22) follows for isotropic distribution of $E_{i}$. [It is in fact not essential to choose $\mathbf{x}_{\mathbf{0}}=0$, since the dependence on the base point $\mathbf{x}_{\mathbf{0}}$ will drop out from all physical quantities. All physical observables, as we shall see, depend only on the difference $A^{+}(\mathbf{x})-A^{+}(\mathbf{y})$ of the vector potentials.] In this leading logarithmic approximation, the dipole scattering amplitude of Eq. (2.20) is

$$
\left\langle\left\langle\frac{1}{N} \operatorname{Tr}\left[W^{F \dagger}(\mathbf{x}) W^{F}(\mathbf{y})\right]\right\rangle\right\rangle=\exp \left[-\frac{(\mathbf{x}-\mathbf{y})^{2}}{8} Q_{s}^{2}\right],
$$

with the saturation momentum

$$
Q_{s}^{2}=4 C_{F} \int d x^{-} \mu^{2}\left(x^{-}\right) .
$$

Thus, to reiterate, the procedure of calculating any observable in the eikonal approximation is the following. For a given initial state, we calculate its eikonal propagation according to Eq. (2.2). Next we "project out" the subspace which is spanned by the possible initial states, according to Eq. (2.9). Then we calculate the observable as in Eq. (2.11). Then we perform the averaging over the initial state if this is needed-for example the averaging over the initial polarization in the case of unpolarized scattering. And finally we average over the target field ensemble in the two gluon exchange approximation with the only nontrivial target average specified in Eq. (2.18) [and Eq. (2.22)].

\section{With due respect to the target}

In the eikonal scheme described above, one does not follow the details of the dynamics on the target side, since the target is modeled by a field distribution rather than being assigned a wave function of its own. As a result, the inelastic cross section (2.8) calculated in this way is underestimated since it accounts only for processes in which the projectile scatters inelastically. Other inelastic processes in the usual sense, in which e.g. the projectile remains intact but the target breaks up, are not included in $\sigma_{\text {inel }}^{\text {proj }}$.

In the same way, the cross section $\sigma_{\mathrm{el}}^{\mathrm{proj}}$ in Eq. (2.15) accounts for the probability that the projectile undergoes elastic scattering. Thus its definition includes the probability of inelastic processes where only the target is excited. Such "diffractive" processes are correctly included in the diffractive cross section $\sigma_{\text {diff }}$ in Eq. (2.17), but in the above, projectile biased discussion, they are shifted from their rightful place in $\sigma_{\text {inel }}$ into $\sigma_{\text {el }}$. As we shall see in our explicit calculations, these contributions are suppressed by powers of $1 / N_{c}$ in the situation where the projectile is much smaller than the target. It is however unclear to us whether the same is true when projectile and target are roughly of the same size.

In order to have the proper separation between elastic and inelastic contributions, we consider now the role of the target wave function. We observe first that the target field averaging described above amounts to calculating averages of the ap- 


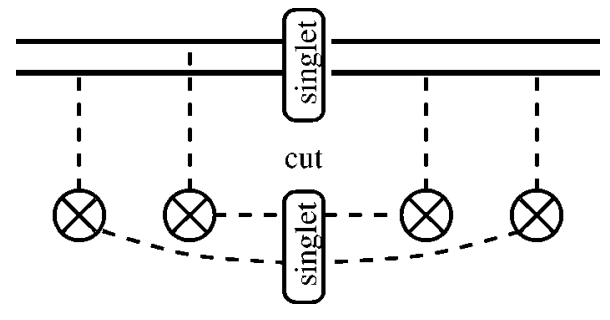

FIG. 2. Schematic picture of a diffractive contribution to the $q \bar{q}$ scattering cross section which does contribute to the inelastic scattering cross section.

propriate Wilson loops in the target wave function. Thus for example

$$
\begin{aligned}
\left\langle\left\langle\operatorname{Tr}\left[W^{F^{\dagger}}(\mathbf{x}) W^{F}(\mathbf{y})\right]\right\rangle\right\rangle & \equiv\left\langle\Psi_{\text {in }}^{t}\left|\operatorname{Tr}\left[W^{F^{\dagger}}(\mathbf{x}) W^{F}(\mathbf{y})\right]\right| \Psi_{\text {in }}^{t}\right\rangle \\
& =\int D A \operatorname{Tr}\left[W^{F^{\dagger}}(\mathbf{x}) W^{F}(\mathbf{y})\right]\left|\psi^{t}[A]\right|^{2},
\end{aligned}
$$

where $\Psi_{\text {in }}^{t}$ is the initial state target wave function which in the gluon field basis has the form

$$
\left|\Psi_{\text {in }}^{t}\right\rangle=\psi^{t}[A]|A\rangle
$$

We can now parallel the discussion of Sec. II A, paying proper attention to the target wave function effects. The initial wave function of the projectile + target system is

$$
\Psi_{\text {in }}=\sum_{\left\{\alpha_{i}, x_{i}\right\}} \psi\left(\left\{\alpha_{i}, x_{i}\right\}\right) \psi^{t}[A]\left|\left\{\alpha_{i}, x_{i}\right\}\right\rangle \otimes|A\rangle
$$

The outgoing wave function is

$$
\begin{aligned}
\Psi_{\text {out }}= & \mathcal{S} \Psi_{\text {in }}=\sum_{\left\{\alpha_{i}, x_{i}\right\}} \psi\left(\left\{\alpha_{i}, x_{i}\right\}\right) \prod_{i} W\left(x_{i}\right)_{\alpha_{i} \beta_{i}} \psi^{t}[A]\left|\left\{\beta_{i}, x_{i}\right\}\right\rangle \\
& \otimes|A\rangle .
\end{aligned}
$$

Although the incoming wave function is a product of the wave functions of the target and the projectile, the outgoing wave function is not factorizable, since the eikonal factors $W$ depend both on the coordinates of the projectile particles and on the target fields. The $S$-matrix element or overlap that determines the inelastic scattering probability (2.4) reads now

$$
s=\left\langle\Psi_{\text {in }} \mid \Psi_{\text {out }}\right\rangle=\left\langle\left[\langle W\rangle_{t}\right]\right\rangle_{p},
$$

where the incoming and outgoing wave functions (2.29) and (2.30) contain both the projectile and the target parts, rather than just the projectile parts as in Eqs. (2.1) and (2.2). Here we denoted the Wilson loop factors arising in the $S$ matrix collectively by $W,\langle\rangle_{t}$ denotes the averaging over the target fields (wave function) as in Eq. (2.27), and \langle\rangle$_{p}$ is the expectation value with respect to the incoming projectile wave function. ${ }^{1}$ The same goes for the elastic probability of Eq. (2.14) and the total probability of Eq. (2.12). Thus we find

$$
\begin{gathered}
P_{\text {inel }}=1-|s|^{2}, \\
P_{\mathrm{el}}=|1-s|^{2}, \\
P_{\mathrm{tot}}=P_{\text {inel }}+P_{\mathrm{el}}=2-s-s^{*}, \\
\sigma_{\text {inel,el,tot }}=\int d^{2} b P_{\text {inel,el,tot }}(b) .
\end{gathered}
$$

The $S$-matrix element $s$, given by Eq. (2.31), already contains the target average.

We therefore see that in order to calculate the true elastic and inelastic cross sections, the "target field averaging" has to be performed on the amplitude level rather than on the cross section level as is sometimes thought [20]. The difference between these averaging procedures has a clear physical meaning: they differ in the treatment of the diffractive states close to the rapidity of the target (see Fig. 2).

Not all quantities are affected by this more careful treatment of the target wave function in calculating the overlap (2.31). The total cross section is not sensitive to this, since it is linear in $s$ and therefore contains in any case only one averaging over the target fields. Physically this is because it does not care about whether we assign the target side diffractive contribution to the elastic or the inelastic probability. The diffractive cross section is not affected since we defined diffractive processes as those in which the projectile wave function emerges from the interaction region in a color singlet state. This definition does not refer to the final state of the target, and thus the target averaging should only be performed once, on the cross section level. The number of produced gluons is also unaffected if we are interested in gluons at rapidities far from those of the target.

In the rest of this paper, we apply the eikonal approximation to a variety of simple cases. Whenever we are interested in the cross sections, we will calculate them according to both definitions: the one referring only to the projectile as described in Sec. II A and the one including the proper treatment of the target wave function, as described above. The projectile biased quantities we will denote by the superscript "proj". We calculate both quantities in order to illustrate the differences between them. We stress, however, that the physical inclusive cross sections are given by Eq. (2.35). In the eikonal approximation, the proper calculation of the elastic and inelastic cross section involves the target field averages on the amplitude level.

\footnotetext{
${ }^{1}$ Here, in order to project out the projectile states with $p_{\gamma}$, we proceed in exactly the same way as explained in Eqs. (2.9),(2.10). We thus assume that the only degrees of freedom that characterize the target are the vector potentials, and that there is therefore no need to perform an analogous projection on the target wave function.
} 


\section{GLUON PRODUCTION}

As a first illustration of our formalism, we present a toy calculation of the number of gluons produced in the high energy scattering of a quark off a nuclear target.

\section{A. Gluon production in $q+A$}

The incoming state in this case is a single quark idealized as the Fock state $|\alpha\rangle$ of the bare quark, supplemented by the coherent state of quasireal gluons which build up the Weizsäcker-Williams field $f(\mathbf{x})$. This can be written as a coherent state of a gluon field - the result of evolving the bare quark state by free evolution from time $-\infty$ to the moment of the interaction:

$$
\begin{aligned}
U_{-}|\alpha\rangle= & \exp \left(-\int d \mathbf{x}|f(\mathbf{x})|^{2}+i \int d \mathbf{x} d \xi f(\mathbf{x})\left[a_{d}(\mathbf{x}, \xi)\right.\right. \\
& \left.\left.+a_{d}^{\dagger}(\mathbf{x}, \xi)\right] T^{d}\right)|\alpha\rangle \\
\approx & |\alpha\rangle+\int d \mathbf{x} d \xi f(\mathbf{x}) T_{\alpha \beta}^{b}|\beta ; b(\mathbf{x}, \xi)\rangle .
\end{aligned}
$$

Here, $U_{-}$denotes the unitary free time evolution from $-\infty$ up to the target, $a_{d}(\mathbf{x}, \xi), a_{d}^{\dagger}(\mathbf{x}, \xi)$ are the annihilation and creation operators for gluons of color $d$ at transverse position $\mathbf{x}$, and rapidity $\xi$, and $T^{d}$ are generators of the fundamental representation of $S U(N)$. Lorentz and spin indices are suppressed. We work in the light cone gauge appropriate to the projectile, that is $A^{-}=0$. In this gauge the gluon field of the projectile is the WeizsäckerWilliams field

$$
A^{i}(\mathbf{x}) \propto \theta\left(x^{-}\right) f_{i}(\mathbf{x}), \quad f_{i}\left(x_{\perp}\right) \propto g \frac{\mathbf{x}_{i}}{\mathbf{x}^{2}}
$$

where $x^{-}=0$ is the light cone coordinate of the quark in the wave function. The integration over the rapidity of the gluon in the wave function (3.1) goes over the gluon rapidities smaller than that of the quark. In the following we will suppress the rapidity label. We are interested in calculating the distribution of produced gluons per unit rapidity and since the wave function does not depend on rapidity, this distribution is obviously flat.

The Weizsäcker-Williams field is proportional to the QCD coupling and is considered to be small. The higher powers of $|f(\mathbf{x})|^{2}$ are thus negligible and the approximation (3.1) applies.

The interaction $S$ of the projectile wave function (3.1) with the target results in the phase shifts described by eikonal Wilson lines,

$$
\begin{aligned}
\Psi_{\text {out }}=S U_{-}|\alpha\rangle= & W_{\alpha \gamma}^{F}(\mathbf{0})|\gamma\rangle+\int d \mathbf{x} f(\mathbf{x}) T_{\alpha \beta}^{b} \\
& \times W_{\beta \gamma}^{F}(\mathbf{0}) W_{b c}^{A}(\mathbf{x})|\gamma ; c(\mathbf{x})\rangle .
\end{aligned}
$$

Here $W^{F}(\mathbf{0})$ and $W^{A}(\mathbf{x})$ are the Wilson lines in the fundamental and adjoint representations respectively, corresponding to the propagating quark at the transverse position $\mathbf{x}_{q}$ $=\mathbf{0}$ and gluon at $\mathbf{x}_{g}=\mathbf{x}$.

Gluon production being an inelastic process, we have to project out of the outgoing wave function the component lying in the subspace spanned by the incoming states with arbitrary color orientation $\alpha$. Thus the index $\gamma$ in the projection operator (2.10) has here the meaning of the quark color index:

$$
\left|\delta \Psi_{\alpha}\right\rangle=U_{+}\left[\left|\Psi_{\text {out }}\right\rangle-\sum_{\gamma} U_{-}|\gamma\rangle\left\langle\gamma U_{-}^{\dagger} U_{+}^{\dagger} \mid \Psi_{\text {out }}\right\rangle\right] .
$$

To lowest order in $f^{2}$ only the no-gluon component of $\Psi_{\text {out }}$ contributes in the last (overlap) term:

$$
\begin{aligned}
& \left|\delta \Psi_{\alpha}\right\rangle=U_{+} \int d \mathbf{x} f(\mathbf{x})\left[T_{\alpha \beta}^{b} W_{\beta \gamma}^{F}(\mathbf{0}) W_{b c}^{A}(\mathbf{x})-T_{\beta \gamma}^{c} W_{\alpha \beta}^{F}(\mathbf{0})\right] \\
& \times|\gamma ; c(\mathbf{x})\rangle
\end{aligned}
$$

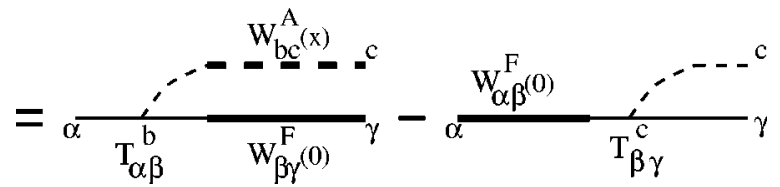

As illustrated by the diagrammatic representation of this equation, the structure of the second term corresponds precisely to "emission after interaction". By this we mean that the gluon is emitted from the initial quark state rotated by the eikonal factor $W^{F}$, while there is no eikonal factor $W^{A}$ that accompanies the gluon propagating through the target. Thus this term is the exact counterpart of the Feynman diagrams containing gluon emission from final state quark. Another point to note is that since the right-hand side (RHS) of Eq. (3.5) has an explicit factor $f$, to lowest order in $f^{2}$ the evolution operator $U_{+}$in Eq. (3.5) has to be approximated by unity [cf. Eq. (3.1)], as noted in the previous section.

The number spectrum of produced gluons is obtained from Eq. (3.5) by calculating the expectation value of the number operator in the state $\delta \Psi_{\alpha}$, averaged over the incoming color index $\alpha$. After some color algebra (see Appendix B for technical details) we obtain 


$$
\begin{aligned}
N_{\text {prod }}(\mathbf{k})= & \frac{1}{N} \sum_{\alpha}\left\langle\delta \Psi_{\alpha}\left|a_{d}^{\dagger}(\mathbf{k}) a_{d}(\mathbf{k})\right| \delta \Psi_{\alpha}\right\rangle \\
= & C_{F} \int d \mathbf{x} d \mathbf{y} e^{i \mathbf{k} \cdot(\mathbf{x}-\mathbf{y})} f(\mathbf{x}) f(\mathbf{y}) \\
& \times\left[1-\frac{1}{N^{2}-1}\left\langle\left\langle\operatorname{Tr}\left[W^{A \dagger}(\mathbf{x}) W^{A}(\mathbf{0})\right]\right\rangle\right\rangle_{t}\right. \\
& -\frac{1}{N^{2}-1}\left\langle\left\langle\operatorname{Tr}\left[W^{A \dagger}(\mathbf{y}) W^{A}(\mathbf{0})\right]\right\rangle\right\rangle_{t} \\
& \left.+\frac{1}{N^{2}-1}\left\langle\left\langle\operatorname{Tr}\left[W^{A \dagger}(\mathbf{y}) W^{A}(\mathbf{x})\right]\right\rangle\right\rangle_{t}\right] .
\end{aligned}
$$

Here, all the information about the scattering properties of the target is encoded in the target average of the product of adjoint Wilson loops which are determined by Eqs. (2.18) and (2.22). The explicit calculation of this as well as some more complicated Wilson loop averages is given in Appendix $\mathrm{C}$. The result is

$$
\frac{1}{N^{2}-1}\left\langle\left\langle\operatorname{Tr}\left[W^{A \dagger}(\mathbf{y}) W^{A}(\mathbf{x})\right]\right\rangle\right\rangle_{t}=\exp \left[-C_{A} v(\mathbf{x}-\mathbf{y})\right]
$$

or, in the leading logarithmic approximation

$$
\exp \left[-\frac{(\mathbf{x}-\mathbf{y})^{2}}{8} \frac{C_{A}}{C_{F}} Q_{s}^{2}\right]
$$

With the explicit form of the Weizsäcker-Williams field of the quark projectile in configuration space,

$$
C_{F} f(\mathbf{x}) f(\mathbf{y})=\left\langle A_{p}(x) A_{p}(y)\right\rangle_{\mathrm{proj}}=\frac{\alpha_{s} C_{F}}{2 \pi} \frac{\mathbf{x} \cdot \mathbf{y}}{\mathbf{x}^{2} \mathbf{y}^{2}}
$$

the produced number spectrum (3.6) for $q+A$ coincides with the expression given by Kovchegov and Mueller [5]. Fourier transforming Eq. (3.6), we see that it is nothing but the Gunion-Bertsch gluon radiation cross section [23] for a hard quark which receives a transverse momentum transfer $\mathbf{k}_{x}$ distributed with Gaussian probability distribution:

$$
\begin{aligned}
N_{\text {prod }}(\mathbf{k})= & \int d \mathbf{x} d \mathbf{y} e^{i k(x-y)} \frac{\mathbf{x} \cdot \mathbf{y}}{\mathbf{x}^{2} \mathbf{y}^{2}}\left(1+e^{-(\mathbf{x}-\mathbf{y})^{2}\left(C_{A} / 8 C_{F}\right) Q_{s}^{2}}\right. \\
& -e^{-\mathbf{x}^{2}\left(C_{A} / 8 C_{F}\right) Q_{s}^{2}-e^{\left.-\mathbf{y}^{2}\left(C_{A} / 8 C_{F}\right) Q_{s}^{2}\right)}} \\
= & \frac{8 \pi C_{F}}{C_{A}} \frac{1}{Q_{s}^{2}} \int d \mathbf{k}_{x} e^{\left(-2 C_{F} / C_{A} Q_{s}^{2}\right) \mathbf{k}_{x}^{2}} \frac{\mathbf{k}_{x}^{2}}{\mathbf{k}^{2}\left(\mathbf{k}_{x}-\mathbf{k}\right)^{2}} .
\end{aligned}
$$

\section{B. Gluon production in $\pi(q \bar{q})+A$}

As a second example, we consider for the initial state wave function a quark-antiquark pair which may be viewed as a simplified representation of a hadron. We take the quark to be at the transverse position $\mathbf{x}$ and the antiquark at $\mathbf{y}$. The state incoming into the interaction region is

$$
\begin{aligned}
U_{-}|(\alpha, \mathbf{x})(\bar{\alpha}, \mathbf{y})\rangle \approx & |(\alpha, \mathbf{x})(\bar{\alpha}, \mathbf{y})\rangle+\int d \mathbf{z}\left[f(\mathbf{z}-\mathbf{x}) T_{\alpha \beta}^{b} \delta_{\alpha \bar{\beta}}^{-}\right. \\
& \left.-f(\mathbf{z}-\mathbf{y}) \delta_{\alpha \beta} T_{\bar{\beta}}^{b} \bar{\alpha}\right]|(\beta, \mathbf{x})(\bar{\beta}, \mathbf{y})(b, \mathbf{z})\rangle .
\end{aligned}
$$

Here, the color indices of the quark and antiquark are independent; i.e., the incoming quark and antiquark are not taken to be in a global color singlet state. The reason for this is the following. Any realistic hadron wave function apart from a quark and an antiquark has a large number of soft gluons. Those are not the Weizsäcker-Williams (WW) gluons present in Eq. (3.11) but rather genuine soft non-perturbative glue. They do not scatter eikonally since their energy is small. One can reasonably expect that they are completely absorbed by the target. This absorption does not produce any gluons in the central rapidity region which we are attempting to calculate here. However, the presence of these soft gluons serves to randomize the color orientation of $q$ and $\bar{q}$. We thus model the hadronic state as having all color orientations of individual quark and antiquark as equally probable. The implication of this is that the projection operator, which we employ to project out the component of the incoming hadron from the outgoing state, has independent summations over the quark and antiquark color indices.

The outgoing state is

$$
\begin{aligned}
\Psi_{\text {out }}= & W_{\alpha \gamma}^{F}(\mathbf{x}) W_{\gamma \alpha}^{F \dagger}(\mathbf{y})|(\gamma, \mathbf{x})(\bar{\gamma}, \mathbf{y})\rangle+\int d \mathbf{z}\left[f(\mathbf{z}-\mathbf{x}) T_{\alpha \beta}^{a}\right. \\
& \times W_{\beta \gamma}^{F}(\mathbf{x}) W_{\bar{\gamma} \alpha}^{F \dagger}(\mathbf{y}) W_{a b}^{A}(\mathbf{z})-f(\mathbf{z}-\mathbf{y}) T_{\bar{\beta}}^{a}-W_{\alpha \gamma}^{F}(\mathbf{x}) \\
& \left.\times W_{\bar{\gamma} \bar{\beta}}^{F \dagger}(\mathbf{y}) W_{a b}^{A}(\mathbf{z})\right]|(\gamma, \mathbf{x})(\bar{\gamma}, \mathbf{y})(b, \mathbf{z})\rangle
\end{aligned}
$$

and, after projecting out the initial state component,

$$
\begin{aligned}
\delta \Psi_{\alpha \bar{\alpha}}= & \int d \mathbf{z}\left[f(\mathbf{z}-\mathbf{x}) T_{\alpha \beta}^{a} W_{\beta \gamma}^{F}(\mathbf{x}) W_{\bar{\gamma}}^{F \dagger}(\mathbf{y}) W_{a b}^{A}(\mathbf{z})\right. \\
& -f(\mathbf{z}-\mathbf{y}) T_{\bar{\beta}-\bar{\alpha}}^{a} W_{\alpha \gamma}^{F}(\mathbf{x}) W_{\bar{\gamma} \bar{\beta}}^{F \dagger}(\mathbf{y}) W_{a b}^{A}(\mathbf{z}) \\
& -f(\mathbf{z}-\mathbf{x}) T_{\beta \gamma}^{b} W_{\alpha \beta}^{F}(\mathbf{x}) W_{\bar{\gamma}}^{F^{\dagger}}(\mathbf{y})+f(\mathbf{z}-\mathbf{y}) \\
& \left.\times T_{\gamma \bar{\beta}}^{b} W_{\alpha \gamma}^{F}(\mathbf{x}) W_{\bar{\beta} \bar{\alpha}}^{F \dagger}(\mathbf{y})\right]|(\gamma, x)(\bar{\gamma}, y)(b, \mathbf{z})\rangle .
\end{aligned}
$$

Here again we have kept only terms of the lowest order in $f^{2}$. The number of produced gluons is calculated in the state $\delta \Psi$ independently averaging over the initial state color indices $\alpha$ and $\bar{\alpha}$. After some color algebra we get 


$$
\begin{aligned}
N_{\text {prod }}(\mathbf{k})= & \frac{1}{N^{2}} \sum_{\alpha \bar{\alpha}}\left\langle\delta \Psi_{\alpha \bar{\alpha}}\left|a_{d}^{\dagger}(\mathbf{k}) a_{d}(\mathbf{k})\right| \delta \Psi_{\alpha \bar{\alpha}}\right\rangle \\
= & C_{F} \int d \mathbf{z} d \overline{\mathbf{z}} e^{i \mathbf{k} \cdot(\mathbf{z}-\overline{\mathbf{z}})}[f(\mathbf{z}-\mathbf{x}) f(\overline{\mathbf{z}}-\mathbf{x}) \\
& \times\left(1+e^{-N v(\mathbf{z}-\overline{\mathbf{z}})}-e^{-N v(\mathbf{z}-\mathbf{x})}-e^{-N v(\overline{\mathbf{z}}-\mathbf{x})}\right) \\
& +f(\mathbf{z}-\mathbf{y}) f(\overline{\mathbf{z}}-\mathbf{y})\left(1+e^{-N v(\mathbf{z}-\overline{\mathbf{z}})}-e^{-N v(\mathbf{z}-\mathbf{y})}\right. \\
& \left.\left.-e^{-N v(\overline{\mathbf{z}}-\mathbf{y})}\right)\right]
\end{aligned}
$$

In comparison with the number spectrum (3.6), Eq. (3.14) is the independent sum of the radiation spectra for two quarknucleus collisions, with quarks at transverse positions $\mathbf{x}$ and $\mathbf{y}$ respectively. (This can be seen by using $v(\mathbf{x}-\mathbf{y})=[(\mathbf{x}$ $\left.-\mathbf{y})^{2} / 8 C_{F}\right] Q_{s}^{2}$. ) In this sense, it appears sufficient to calculate the $q$ - $A$ gluon number spectrum (3.6) in order to determine the $h-A$ number spectrum for "hadrons" $h$. However, there are at least two obvious limitations to this simplified treatment: first, if color correlations between the projectile partons become important, the gluon emission off different quarks is not independent any more. This will be seen in our discussion of gluon production off a color singlet $q \bar{q}$ Fock state in Sec. IV. Second, if the color charge density in the projectile is large, then its WW field is not an incoherent superposition of the WW fields of its constituents. This in particular is important when both colliding objects are nuclei, as in [16]. We plan to address this question in a future publication [19].

\section{SMALL $x$ DIS IN THE TARGET REST FRAME}

In Sec. III B, we considered the propagation and accompanying gluon radiation of a quark-antiquark pair in a nuclear target without requiring that this color dipole is in a global color singlet state. We argued that this is a simple ansatz for a meson-nucleus collision where the hard quark and antiquark projectile components are not required to be in a singlet state since soft non-perturbative gluon fields are present as well. The situation is different for many problems in small $x$ DIS. In the nuclear target rest frame, the process of interest here is the eikonal propagation of the hadronic Fock components of the virtual photon through the nuclear target field. Because of formation time arguments, these Fock states cannot develop a soft color-carrying field prior to the interaction. The hadronic wave function of the virtual photon is hence perturbative, and the lowest lying $q \bar{q}$ and $q \bar{q} g$ Fock states of the incoming virtual photon are in a color singlet. At not too low values of $x=x_{0}$ the photon wave function has only the $q \bar{q}$ component:

$$
\left|\gamma^{*}\right\rangle=\int d^{2}(\mathbf{x}-\mathbf{y}) d z \psi(\mathbf{x}-\mathbf{y}, z) \frac{1}{\sqrt{N}} \delta_{\alpha \bar{\alpha}}|\alpha(\mathbf{x}), \bar{\alpha}(\mathbf{y}), z\rangle
$$

Here as previously $\mathbf{x}$ and $\mathbf{y}$ are transverse coordinates of the quark and antiquark respectively and $z$ is the fraction of the longitudinal momentum carried by the quark. The explicit form of the wave function $\psi$ for both transverse and longitudinal photons is given in many places (e.g. Ref. [20]) and will not concern us here. We will also not indicate the $z$ dependence of the Fock states explicitly in the following, since the scattering amplitude of the $q \bar{q}$ pair in the eikonal approximation does not depend on $z$.

The DIS cross section is determined by the total cross section of the $q \bar{q}$ pair on the target. The reason it is the total and not the inelastic probability that is important, is because even if only the phase of the $q \bar{q}$ state is changed in the scattering event, the coherence between the components of the $\gamma^{*}$ wave function is disturbed and thus what emerges from the interaction region is some hadronic state. The DIS cross section is thus given by

$$
\sigma^{D I S}=\int d^{2} \mathbf{x} d^{2} \mathbf{y} d z \psi(\mathbf{x}-\mathbf{y}, z) \psi^{*}(\mathbf{x}-\mathbf{y}, z) P_{\text {tot }}^{q \bar{q}}(\mathbf{x}, \mathbf{y}) .
$$

At lower values of $x$ the virtual photon wave function develops a $q \bar{q} g$ Fock space component, due to the WeizsäckerWilliams field of the quark and the antiquark. Thus the wave function of the $q \bar{q}$ pair in Eq. (4.1) is substituted by the normalized state

$$
\begin{aligned}
\frac{1}{\sqrt{N}} \delta_{\alpha \bar{\alpha}}|\alpha(\mathbf{x}), \bar{\alpha}(\mathbf{y})\rangle \rightarrow \Psi_{\text {in }} \\
=\frac{\mathcal{N}}{\sqrt{N}} \delta_{\alpha \bar{\alpha}}|\alpha(\mathbf{x}), \bar{\alpha}(\mathbf{y})\rangle+\frac{1}{\sqrt{N}} \int_{\ln 1 / x_{0}}^{\ln 1 / x} d \xi \int d^{2} \mathbf{z} \\
\quad \times[f(\mathbf{z}-\mathbf{x})-f(\mathbf{z}-\mathbf{y})] T_{\alpha \beta}^{a}|(\beta, \mathbf{x})(\alpha, \mathbf{y})(a, \mathbf{z}, \xi)\rangle,
\end{aligned}
$$

where in the leading logarithmic approximation we have taken the gluon emission amplitude to be independent of the gluon rapidity $\xi$. This wave function is normalized to unity via the factor

$$
\begin{aligned}
\mathcal{N} & =\frac{1}{\sqrt{\left[1+C_{F} \int d^{2} \mathbf{z} F^{2}(\mathbf{x}, \mathbf{y}, \mathbf{z}) \ln \left(x_{0} / x\right)\right]}} \\
& \approx 1-\frac{C_{F}}{2} \int d^{2} \mathbf{z} F^{2}(\mathbf{x}, \mathbf{y}, \mathbf{z}) \ln \left(x_{0} / x\right) .
\end{aligned}
$$

Here and in what follows, we use the following notational shorthand for the Weizsäcker-Williams fields:

$$
F \equiv f(\mathbf{z}-\mathbf{x})-f(\mathbf{z}-\mathbf{y})
$$

The wave function (4.4) is correct for Weizsäcker-Williams photons with rapidity lower than that of both, the quark and 
the antiquark. The photons with intermediate rapidity can be emitted only by the fastest parton (be it $q$ or $\bar{q}$ ). In the leading logarithmic approximation, however, it is the soft photons that dominate the phase space and we only keep those in our analysis.

Even though only $P_{\text {tot }}^{q \bar{q}}$ is relevant for inclusive DIS cross section, in the rest of this section we will discuss also the inelastic and diffractive cross sections of the $q \bar{q}$ scattering on the nuclear target. Those have distinct physical meaning and are useful for calculations of less inclusive quantities. Our starting point is the virtual photon component (4.3). The interaction with the target evolves its wave function into

$$
\begin{aligned}
\Psi_{\text {out }}= & S \Psi_{\text {in }} \\
= & \frac{\mathcal{N}}{\sqrt{N}}\left[W^{F^{\dagger}}(\mathbf{y}) W^{F}(\mathbf{x})\right]_{\bar{\alpha} \alpha}|\alpha(\mathbf{x}), \bar{\alpha}(\mathbf{y})\rangle \\
& +\frac{1}{\sqrt{N}} F\left[W^{F \dagger}(\mathbf{y}) T^{a} W^{F}(\mathbf{x})\right]_{\bar{\alpha} \alpha} \\
& \times W_{a b}^{A}(\mathbf{z})|\alpha(\mathbf{x}), \bar{\alpha}(\mathbf{y}), b(\mathbf{z})\rangle,
\end{aligned}
$$

where we have suppressed the integration over the gluon rapidity.

\section{A. $q \bar{q}$ contribution to photoabsorption cross sections}

We start with the discussion of the contributions from the lowest lying $q \bar{q}$ Fock state. Details of the medium average as well as more differential cross sections are listed in the appendices.

The total scattering probability, which determines the inclusive DIS cross section according to Eq. (4.2), reads

$$
\begin{aligned}
P_{\mathrm{tot}}^{q \bar{q}}= & \left\langle\left\langle 2-\frac{1}{N} \operatorname{Tr}\left[W^{F}(\mathbf{x}) W^{F \dagger}(\mathbf{y})\right]\right.\right. \\
& \left.\left.-\frac{1}{N} \operatorname{Tr}\left[W^{F}(\mathbf{y}) W^{F^{\dagger}}(\mathbf{x})\right]\right\rangle\right\rangle \\
= & 2\left[1-e^{-C_{F} v(\mathbf{x}-\mathbf{y})}\right] .
\end{aligned}
$$

For the inelastic photoabsorption cross section, we get

$$
\begin{aligned}
P_{\text {inel }}^{q \bar{q}} & =1-\frac{1}{N^{2}}\left\langle\left\langle\operatorname{Tr}\left[W^{F \dagger}(\mathbf{x}) W^{F}(\mathbf{y})\right]\right\rangle\right\rangle\left\langle\left\langle\operatorname{Tr}\left[W^{F^{\dagger}}(\mathbf{y}) W^{F}(\mathbf{x})\right]\right\rangle\right\rangle \\
& =1-e^{-2 C_{F} v(\mathbf{x}-\mathbf{y})}
\end{aligned}
$$

$$
\begin{aligned}
P_{\text {inel }}^{q \bar{q} \text { proj }}= & \left\langle\left\langle 1-\frac{1}{N^{2}} \operatorname{Tr}\left[W^{F^{\dagger}}(\mathbf{x}) W^{F}(\mathbf{y})\right]\right.\right. \\
& \left.\left.\times \operatorname{Tr}\left[W^{F \dagger}(\mathbf{y}) W^{F}(\mathbf{x})\right]\right\rangle\right\rangle \\
= & \left\langle\left\langle\frac{N^{2}-1}{N^{2}}-\frac{1}{N^{2}} W_{a b}^{A}(\mathbf{x}) W_{a b}^{A}(\mathbf{y})\right\rangle\right\rangle \\
= & \frac{N^{2}-1}{N^{2}}\left[1-e^{-N v(\mathbf{x}-\mathbf{y})}\right] .
\end{aligned}
$$

As explained in Sec. II, the difference between these two expressions is due to the target side diffractive processes which are not included in Eq. (4.9) but are accounted for in Eq. (4.8). Comparing Eqs. (4.8) and (4.9) we see that this difference is of the order $O\left(1 / N^{2}\right)$. The diffractive probability reads

$$
\begin{aligned}
P_{\mathrm{diff}}^{q \bar{q}}= & \left\langle\left\langle\left(\frac{1}{N} \operatorname{Tr}\left[W^{F}(\mathbf{x}) W^{F^{\dagger}}(\mathbf{y})\right]-1\right)\right.\right. \\
& \left.\left.\times\left(\frac{1}{N} \operatorname{Tr}\left[W^{F}(\mathbf{y}) W^{F^{\dagger}}(\mathbf{x})\right]-1\right)\right\rangle\right\rangle \\
= & \left\langle\left\langle\frac{1}{N^{2}}\left[1+W_{a b}^{A}(\mathbf{x}) W_{a b}^{A}(\mathbf{y})\right]-\frac{1}{N} \operatorname{Tr}\left[W^{F}(\mathbf{x}) W^{F^{\dagger}}(\mathbf{y})\right]\right.\right. \\
& \left.\left.-\frac{1}{N} \operatorname{Tr}\left[W^{F}(\mathbf{y}) W^{F \dagger}(\mathbf{x})\right]+1\right\rangle\right) \\
= & \left(\frac{N^{2}+1}{N^{2}}-2 e^{-C_{F} v(\mathbf{x}-\mathbf{y})}+\frac{N^{2}-1}{N^{2}} e^{-N v(\mathbf{x}-\mathbf{y})}\right) \\
= & \left(1-e^{-C_{F} v(\mathbf{x}-\mathbf{y})}\right)^{2}+O\left(\frac{1}{N^{2}}\right) .
\end{aligned}
$$

The leading $O(1 / N)$ contribution in Eq. (4.10) is the result given by Buchmüller et al. for the diffractive photoabsorption probability [3]. It is easy to see from Eqs. (4.8), (4.7), (2.35) that this contribution is precisely equal to the elastic probability $P_{\mathrm{el}}$, since $P_{\mathrm{el}}=P_{\mathrm{tot}}-P_{\text {inel }}$. This leading $O(1 / N)$ result was later rederived in Ref. [20] and it lead Kovchegov and McLerran to conjecture that the diffractive cross section can be calculated by the procedure of averaging over the target fields on the amplitude level. Physically, however, as noted in Ref. [20] and explained above, such a procedure corresponds to (quasi)elastic scattering where each target color source (nucleon) in the target nucleus remains in a color singlet state. In contrast, the diffractive production requires the outgoing projectile wave function to be in the color singlet state but it can leave some target sources in the color octet and thus the whole nucleus in an excited state (the overall color neutrality of the target is of course still preserved). Our derivation makes it clear that for the diffractive

and 
cross section the averaging must be done on the level of the cross section. We also see that the higher order in $1 / N$ corrections have a clear physical meaning in this case-they distinguish between the diffractive and the (quasi)elastic production.

As our previous discussion suggests the difference between the diffractive and elastic cross sections for the scattering of $q \bar{q}$ pair is due to precisely the same physical final states as the difference between $P_{\text {inel }}$ and $P_{\text {inel }}^{\text {proj }}$. Indeed subtracting from the total cross section (4.7) the projectile biased inelastic one (4.9), one obtains the diffractive cross section (4.10). For more complicated projectile configurations this is not necessarily the case, since the global color is not the only degree of freedom that characterizes the outgoing state. This is also not true beyond the eikonal approximation where the transverse positions of the partons in the projectile may change during the interaction time [24].

\section{B. $q \bar{q} g$ contributions to photoabsorption cross sections}

The non-Abelian Weizsäcker-Williams field in the virtual photon wave function (4.3) is perturbatively small. In the calculations of the last subsection, this was used to neglect all powers of $F$. Now, we discuss the $O\left(F^{2}\right)$ corrections to these photoabsorption cross sections. There are several motivations to do so. Though nominally suppressed by powers of the strong coupling constant, such radiative corrections are known to give important contributions to the leading twist photoabsorption cross sections in some regions of phase space. Moreover, the gluon radiation off the $q \bar{q}$ Fock state can be viewed as a leading contribution to the small $x$ evolution equations. This we shall discuss in Sec. V. The $O\left(F^{2}\right)$ corrections arise from the $q \bar{q} g$ components of the ingoing and outgoing $\gamma^{*}$ wave functions (4.3), (4.4), and (4.6).

The total scattering probability reads

$$
\begin{aligned}
P_{\mathrm{tot}}^{(q \bar{q}+q \bar{q} g)}= & \left\langle\left\langle\left(2-\frac{1}{N} \operatorname{Tr}\left[W^{F \dagger}(\mathbf{x}) W^{F}(\mathbf{y})\right]-\frac{1}{N} \operatorname{Tr}\left[W^{F^{\dagger}}(\mathbf{y}) W^{F}(\mathbf{x})\right]\right)\right\rangle\right. \\
& +\ln \left(x_{0} / x\right) F^{2}\left\langle\left\langle\left\{\frac{1}{2} \operatorname{Tr}\left[W^{F \dagger}(\mathbf{x}) W^{F}(\mathbf{y})\right]+\frac{1}{2} \operatorname{Tr}\left[W^{F \dagger}(\mathbf{y}) W^{F}(\mathbf{x})\right]\right.\right.\right. \\
& \left.\left.\left.-\frac{1}{2 N} \operatorname{Tr}\left[W^{F^{\dagger}}(\mathbf{z}) W^{F}(\mathbf{x})\right] \operatorname{Tr}\left[W^{F \dagger}(\mathbf{y}) W^{F}(\mathbf{z})\right]-\frac{1}{2 N} \operatorname{Tr}\left[W^{F \dagger}(\mathbf{x}) W^{F}(\mathbf{z})\right] \operatorname{Tr}\left[W^{F \dagger}(\mathbf{z}) W^{F}(\mathbf{y})\right]\right\}\right\rangle\right) \\
= & \left(2-2 e^{-C_{F} v(\mathbf{x}-\mathbf{y})}\right)+2 C_{F} \ln \left(x_{0} / x\right) F^{2}\left(e^{-C_{F} v(\mathbf{x}-\mathbf{y})}-e^{-(N / 2)[v(\mathbf{z}-\mathbf{y})+v(\mathbf{z}-\mathbf{x})]+(1 / 2 N) v(\mathbf{x}-\mathbf{y})}\right) .
\end{aligned}
$$

Here and in the following we use the shorthand

$$
F^{2} \equiv \int d^{2} \mathbf{z} F^{2}(\mathbf{x}, \mathbf{y}, \mathbf{z})[\cdots],
$$

which stands for the operator acting by multiplication with the square of the WW field, and integration over its coordinate. Thus in this and the following expressions the coordinate of the gluon $\mathbf{z}$ is always integrated over.

The inelastic photoabsorption probability reads

$$
\begin{aligned}
P_{\text {inel }}^{(q \bar{q}+q \bar{q} g)}= & 1-\frac{1}{N^{2}}\left\langle\left\langle\operatorname{Tr}\left[W^{F^{\dagger}}(\mathbf{x}) W^{F}(\mathbf{y})\right]\right\rangle\right\rangle\left\langle\left\langle\operatorname{Tr}\left[W^{F^{\dagger}}(\mathbf{y}) W^{F}(\mathbf{x})\right]\right\rangle\right\rangle+\ln \left(x_{0} / x\right) F^{2} \frac{1}{2 N^{2}}\left\langle\left\langle\operatorname{Tr}\left[W^{F^{\dagger}}(\mathbf{y}) W^{F}(\mathbf{x})\right]\right\rangle\right\rangle \\
& \times\left\langle\left\langle\left\{N \operatorname{Tr}\left[W^{F^{\dagger}}(\mathbf{x}) W^{F}(\mathbf{y})\right]-\operatorname{Tr}\left[W^{F^{\dagger}}(\mathbf{x}) W^{F}(\mathbf{z})\right] \operatorname{Tr}\left[W^{F^{\dagger}}(\mathbf{z}) W^{F}(\mathbf{y})\right]\right\}\right\rangle\right\rangle+\ln \left(x_{0} / x\right) F^{2} \frac{1}{2 N^{2}}\left\langle\left\langle\operatorname{Tr}\left[W^{F^{\dagger}}(\mathbf{x}) W^{F}(\mathbf{y})\right]\right\rangle\right\rangle \\
& \times\left\langle\left\langle\left\{N \operatorname{Tr}\left[W^{F^{\dagger}}(\mathbf{y}) W^{F}(\mathbf{x})\right]-\operatorname{Tr}\left[W^{F \dagger}(\mathbf{z}) W^{F}(\mathbf{x})\right] \operatorname{Tr}\left[W^{F^{\dagger}}(\mathbf{y}) W^{F}(\mathbf{z})\right]\right\}\right\rangle\right\rangle \\
= & 1-e^{-2 C_{F} v(\mathbf{x}-\mathbf{y})}+2 C_{F} \ln \left(x_{0} / x\right) F^{2} e^{-C_{F} v(\mathbf{x}-\mathbf{y})}\left(e^{-C_{F} v(\mathbf{x}-\mathbf{y})}-e^{(-N / 2)[v(\mathbf{z}-\mathbf{y})+v(\mathbf{z}-\mathbf{x})]+(1 / 2 N) v(\mathbf{x}-\mathbf{y})}\right)
\end{aligned}
$$

Finally, the diffractive probability reads

$$
\begin{aligned}
P_{\mathrm{diff}}^{(q \bar{q}+q \bar{q} g)}= & \left\langle\left(\left(\frac{1}{N} \operatorname{Tr}\left[W^{F}(\mathbf{x}) W^{F \dagger}(\mathbf{y})\right]-1\right)\left(\frac{1}{N} \operatorname{Tr}\left[W^{F}(\mathbf{y}) W^{F \dagger}(\mathbf{x})\right]-1\right)+C_{F} \ln \left(x_{0} / x\right) F^{2}\left\{-\left(\frac{1}{N} \operatorname{Tr}\left[W^{F}(\mathbf{x}) W^{F \dagger}(\mathbf{y})\right]-1\right)\right.\right.\right. \\
& \times\left(\frac{1}{N} \operatorname{Tr}\left[W^{F}(\mathbf{y}) W^{F^{\dagger}}(\mathbf{x})\right]-1\right)+\left(\frac{1}{N^{2}-1} \operatorname{Tr}\left[W^{F \dagger}(\mathbf{y}) W^{F}(\mathbf{z})\right] \operatorname{Tr}\left[W^{F \dagger}(\mathbf{z}) W^{F}(\mathbf{x})\right]-\frac{1}{N\left(N^{2}-1\right)} \operatorname{Tr}\left[W^{F}(\mathbf{x}) W^{F^{\dagger}}(\mathbf{y})\right]-1\right) \\
& \left.\left.\left.\times\left(\frac{1}{N^{2}-1} \operatorname{Tr}\left[W^{F \dagger}(\mathbf{x}) W^{F}(\mathbf{z})\right] \operatorname{Tr}\left[W^{F \dagger}(\mathbf{z}) W^{F}(\mathbf{y})\right]-\frac{1}{N\left(N^{2}-1\right)} \operatorname{Tr}\left[W^{F}(\mathbf{y}) W^{F \dagger}(\mathbf{x})\right]-1\right)\right\}\right)\right\rangle
\end{aligned}
$$


The explicit averaging of this expression over the target field is calculated in Appendix C. The final result is

$$
\begin{aligned}
P_{\mathrm{diff}}^{(q \bar{q}+q \bar{q} g)}= & \left(\frac{N^{2}+1}{N^{2}}-2 e^{-C_{F} v(\mathbf{x}-\mathbf{y})}+\frac{N^{2}-1}{N^{2}} e^{-N v(\mathbf{x}-\mathbf{y})}\right)+C_{F} \ln \left(x_{0} / x\right) F^{2}\left[-\frac{N^{2}-2}{N^{2}\left(N^{2}-1\right)}+2 e^{-C_{F} v(\mathbf{x}-\mathbf{y})}\right. \\
& -\frac{\left(N^{2}-1\right)^{3}-1}{N^{2}\left(N^{2}-1\right)^{2}} e^{-N v(\mathbf{x}-\mathbf{y})}+\frac{N^{2}-2}{N^{2}\left(N^{2}-1\right)}\left(e^{-N v(\mathbf{z}-\mathbf{x})}+e^{-N v(\mathbf{z}-\mathbf{y})}\right)+\frac{N^{2}-4}{2\left(N^{2}-1\right)} e^{-N[v(\mathbf{z}-\mathbf{x})+v(\mathbf{z}-\mathbf{y})]} \\
& -2 e^{(-N / 2)[v(\mathbf{z}-\mathbf{x})+v(\mathbf{z}-\mathbf{y})]}\left(e^{(1 / 2 N) v(\mathbf{x}-\mathbf{y})}-\frac{2}{N^{2}\left(N^{2}-1\right)} e^{(-N / 2) v(\mathbf{x}-\mathbf{y})}\right) \\
& \left.+\frac{N^{2}}{4\left(N^{2}-1\right)}\left(\frac{N+3}{N+1} e^{-(N+1)[v(\mathbf{z}-\mathbf{x})+v(\mathbf{z}-\mathbf{y})]+v(\mathbf{x}-\mathbf{y})}+\frac{N-3}{N-1} e^{-(N-1)[v(\mathbf{z}-\mathbf{x})+v(\mathbf{z}-\mathbf{y})]-v(\mathbf{x}-\mathbf{y})}\right)\right] .
\end{aligned}
$$

To leading order in $1 / N$ this simplifies to

$$
P_{\text {diff }}^{(q \bar{q}+q \bar{q} g)}=\left(1-e^{(-N / 2) v(\mathbf{x}-\mathbf{y})}\right)^{2}+C_{F} F^{2} \ln \left(x_{0} / x\right)\left\{-\left(1-e^{(-N / 2) v(\mathbf{x}-\mathbf{y})}\right)^{2}+\left(1-e^{(-N / 2)[v(\mathbf{z}-\mathbf{x})+v(\mathbf{z}-\mathbf{y})]}\right)^{2}\right\}+O\left(\frac{1}{N^{2}}\right) .
$$

For completeness, we also give the projectile biased inelastic probability

$$
\begin{aligned}
P_{\text {inel }}^{(q \bar{q}+q \bar{q} g) \text { proj }}= & \left\langle\left\langle 1-\mathcal{N}^{4}\left(\frac{1}{N^{2}}+\frac{1}{N^{2}} W_{a b}^{A}(\mathbf{x}) W_{a b}^{A}(\mathbf{y})\right)-\frac{2}{N^{2}} \ln \left(x_{0} / x\right) F^{2}\left[\frac{1}{2 N} W_{a b}^{A}(\mathbf{z}) W_{a b}^{A}(\mathbf{x})+\frac{1}{2 N} W_{a b}^{A}(\mathbf{z}) W_{a b}^{A}(\mathbf{y})\right.\right.\right. \\
& \left.\left.\left.+\frac{1}{4} W_{a b}^{A}(\mathbf{z}) W_{c d}^{A}(\mathbf{y}) W_{e f}^{A}(\mathbf{x})\left(d_{a c e} d_{b d f}+f_{a c e} f_{b d f}\right)\right]\right\rangle\right) \\
= & \frac{N^{2}-1}{N^{2}}\left(1-e^{-N v(\mathbf{x}-\mathbf{y})}\right)+2 C_{F} \ln \left(x_{0} / x\right) F^{2}\left\{\frac{1}{N^{2}}+\frac{N^{2}-1}{N^{2}} e^{-N v(\mathbf{x}-\mathbf{y})}-\frac{1}{N^{2}} e^{-N v(\mathbf{z}-\mathbf{x})}\right. \\
& \left.-\frac{1}{N^{2}} e^{-N v(\mathbf{z}-\mathbf{y})}-\left(1-\frac{2}{N^{2}}\right) e^{(-N / 2)[v(\mathbf{z}-\mathbf{x})+v(\mathbf{z}-\mathbf{y})+v(\mathbf{x}-\mathbf{y})]}\right\} .
\end{aligned}
$$

To leading order $O(1 / N)$, the $q \bar{q}$ contributions to the projectile biased and true photoabsorption cross sections coincide. However, for the $q \bar{q} g$ configuration, the elastic and the diffractive cross sections do not agree even at the leading order in $1 / N$. The elastic cross section can be immediately inferred from Eq. (4.11). To leading order in $1 / N$,

$$
P_{\mathrm{el}}^{(q \bar{q}+q \bar{q} g)}=\left(1-e^{-(N / 2) v(\mathbf{x}-\mathbf{y})}\right)^{2}+2 C_{F} \ln \left(x_{0} / x\right) F^{2}\left(1-e^{(-N / 2) v(\mathbf{x}-\mathbf{y})}\right)\left(e^{(-N / 2) v(\mathbf{x}-\mathbf{y})}-e^{(-N / 2)[v(\mathbf{z}-\mathbf{y})+v(\mathbf{z}-\mathbf{x})]}\right)+O\left(1 / N^{2}\right) .
$$

This has to be compared with Eq. (4.16). The difference in the $O\left(F^{2}\right)$ terms is due to the fact that as opposed to the $q \bar{q}$ case, the quantum state now is characterized by a new degree of freedom. This degree of freedom is the relative phase between the $q \bar{q}$ and the $q \bar{q} g$ components of the wave function. Thus the outgoing state can be a color singlet which is not identical to the incoming one.

\section{EIKONAL LOW $x$ EVOLUTION}

The results of the previous section allow us to derive evolution equations for inelastic and diffractive DIS cross sections in the eikonal approximation. In the operator form, the equation for the evolution of the inelastic cross section is the same as that of Balitsky [7]. Our derivation is very simple and allows us to trace explicitly some of the effects that limit the range of validity of this equation. We also present the all order $1 / N$ corrections to Kovchegov's version of this evolution equation. Those are obtained with the use of Fierz identities as described in the appendixes. The equation for the diffractive cross section as given here in the operator form is new. In the large $N$ limit it reduces to the equation discussed in Ref. [21] and we present $1 / N$ corrections to this form.

\section{A. Inelastic cross section}

Consider DIS at some low value of $x_{0}$. We work in the frame where the photon wave function at $x_{0}$ has only a $\bar{q} q$ component, but the target ensemble is characterized by large values of the field. There are two distinct physical situations that correspond to this. One is the scattering on a nuclear 
target at not very high energy. In this case the large target fields are due to large number of nucleons in the target rest frame, and no significant $x$ evolution has taken place $\left(x_{0}\right.$ is not very low). Another situation is that the scattering is on a single hadron and $x_{0}$ is very small. In this case the large target fields have been generated due to the low $x$ evolution which in our frame has been put into the target. There are some clear physical differences between the two situations, which we will discuss later, but for now we treat them both in the same framework.

Thus at $x_{0}$ the DIS cross section is given by Eq. (4.2). The first line of Eq. (4.7) then expresses it in terms of the glue fields of the target, while the second line gives its explicit expression in terms of our ansatz for the target field averages. Now consider a lower value of $x$. It is convenient to use the frame where all the evolution between $x_{0}$ and $x$ is occurring in the photon wave function. This means that to achieve higher interaction energy the photon is boosted, while the target remains the same. Thus the target field ensemble is unchanged, but the photon wave function at $x$ has an extra $\bar{q} q g$ component according to Eqs. (4.3) and (4.4). The inelastic scattering probability is now given according to Eq. (4.2) but with $P^{(q \bar{q}+q \bar{q} g)}$ of Eq. (4.11) substituted for $P^{q \bar{q}}$. If we were to put the evolution in the target instead, the inelastic cross section would still be given by Eq. (4.2) but with the average performed over the new target field ensemble. We can thus combine Eqs. (4.7) and (4.11) into a differential equation governing the change of the target field averages with $x$ [we define $\left.\xi \equiv \ln \left(x_{0} / x\right)\right]$ :

$$
\begin{aligned}
-\frac{d}{d \xi} & \left\langle\left\langle\operatorname{Tr}\left[W^{F^{\dagger}}(\mathbf{x}) W^{F}(\mathbf{y})\right]\right\rangle\right\rangle \\
= & F^{2}\left\langle\left(\frac{N}{2} \operatorname{Tr}\left[W^{F^{\dagger}}(\mathbf{x}) W^{F}(\mathbf{y})\right]-\frac{1}{2} \operatorname{Tr}\left[W^{F \dagger}(\mathbf{x}) W^{F}(\mathbf{z})\right]\right.\right. \\
& \left.\left.\times \operatorname{Tr}\left[W^{F^{\dagger}}(\mathbf{z}) W^{F}(\mathbf{y})\right]\right\rangle\right) .
\end{aligned}
$$

This equation is identical to that derived by Balitsky [7]. Taking now the averages over the target fields and denoting the "dipole scattering probability" by

$$
D(\mathbf{x}, \mathbf{y})=1-e^{-C_{F} v(\mathbf{x}, \mathbf{y})},
$$

we have

$$
\begin{aligned}
\frac{d}{d \xi} D(\mathbf{x}, \mathbf{y})= & C_{F} F^{2}\left\{1-D(\mathbf{x}, \mathbf{y})-[1-D(\mathbf{z}, \mathbf{y})]^{N^{2} /\left(N^{2}-1\right)}\right. \\
& \left.\times[1-D(\mathbf{x}, \mathbf{z})]^{N^{2} /\left(N^{2}-1\right)}[1-D(\mathbf{x}, \mathbf{y})]^{-1 /\left(N^{2}-1\right)}\right\} .
\end{aligned}
$$

To leading order in $1 / N$ this reduces to the equation derived by Kovchegov [7].

The extra terms in Eq. (5.3) furnish $1 / N$ corrections to Kovchegov's form of the evolution equation. Interestingly enough the corrections arise as non integer powers of $[1-D]$, and thus at small $D$ correspond to infinite series. In the saturation regime, where $D$ is close to unity the effect of these corrections may very well be large, and it would be quite interesting to explore these effects numerically, similarly to Ref. [25].

These corrections have a very simple meaning. As discussed by Kovchegov [7], the leading 1/N approximation in the present context is equivalent to assuming that the dipoles interact with the target independently. From our derivation of the target averages in Appendix C, it is obvious that the corrections in Eq. (5.3) arise precisely due to the breakdown of the independent propagation beyond the leading order in $1 / N$.

What is meant by "independent propagation"? Physically, as discussed in Sec. II, in the eikonal approximation the partons of the projectile propagate through the target field completely independently of each other. This statement follows from the eikonal kinematics and does not rely on the $1 / N$ expansion. However, the fields in the target are correlated with each other at different transverse coordinates. As a result the eikonal phases picked up by the partons are not independent when averaged over the target field ensemble. If the target fields are small locally at every longitudinal coordinate, so that our model for averaging is applicable, then in the leading order in $1 / N$ all eikonal phase averages factorize into amplitudes corresponding to fundamental dipoles. In this sense, the dipoles "propagate independently" only to leading order in $1 / N$. Moreover, it is crucial for this factorization that any given source in the target can exchange at most two gluons with the projectile. If the fields are locally not small, it is possible that the propagation is still eikonal, but the $1 / N$ factorization breaks down.

We finally discuss the limitations of this approach. There are at least two reasons to expect that the evolution equation derived here breaks down at very low values of $x$ :

Firstly, the derivation assumes that all the evolution up until $x_{0}$ occurred in the target wave function. Further evolution is nothing but boosting of the target. In the boosted frame the gluon fields contract in the longitudinal direction and their amplitude grows, as discussed in Refs. [4,8]. This does not mean that the effective width of the target shrinks, since the low momentum components of the glue field also become important. The effective width of the target then can remain approximately constant, but the fields inside it grow. Therefore, if the target wave function has undergone a lengthy low $x$ evolution, the target fields have significantly increased locally and the averaging procedure employed here and in Ref. [7] breaks down. Thus we expect Eq. (5.3) to be a sensible description for targets which are large but "soft", like a large nucleus in its rest frame, but not for targets which are "hard", like a very energetic proton.

Second, if the evolution is followed to very small values of $x$, the projectile wave function itself becomes dense. Then any further evolution has to take into account that the Weizsäcker-Williams field of the projectile becomes large. Further evolution then becomes nonlinear already on the projectile side, since we would not be able to neglect higher orders of the field $F$ in all our calculations. The onset of this effect may be expected to occur at the scale 


$$
\ln \left(x_{0} / x\right) \simeq \frac{1}{F^{2}}
$$

when the $q \bar{q}$ and $q \bar{q} g$ contributions to the photoabsorption cross section become comparable. In the language of the second paper in Ref. [7], such higher $O\left(F^{2}\right)$ corrections are due to "Pomeron loops." The evolution equation then breaks down already in the operatorial form. Another way of saying it, is that the averages of the products of large number of Wilson lines is not described by a straightforward generalization of Eq. (5.1) given in Ref. [7]. It is an interesting question whether the evolution equation of [8] accounts better for this physics. As discussed in [17], the Jalilian-MarianKovner-Leonidov-Weigert (JKLW) evolution is different form the Balitskii-Kovchegov (BK) evolution, when the fluctuation fields are large. This is precisely the case of a large projectile. However whether this difference is due to different physics content or to technical difficulties with JKLW equation is not clear at the moment, ${ }^{2}$ as discussed in [17].

\section{B. Diffractive cross section}

We can repeat the same exercise for the diffractive cross section. Using Eqs. (4.10) and (4.14) we get

$$
\begin{aligned}
\frac{d}{d \xi} P_{\text {diff }}(x, y)= & -C_{F} F^{2} /\left\langle\left(\frac{1}{N} \operatorname{Tr}\left[W^{F}(\mathbf{x}) W^{F^{\dagger}}(\mathbf{y})\right]-1\right)\left(\frac{1}{N} \operatorname{Tr}\left[W^{F}(\mathbf{y}) W^{F^{\dagger}}(\mathbf{x})\right]-1\right)\right. \\
& -\left(\frac{1}{N^{2}-1} \operatorname{Tr}\left[W^{F^{\dagger}}(\mathbf{y}) W^{F}(\mathbf{z})\right] \operatorname{Tr}\left[W^{F^{\dagger}}(\mathbf{z}) W^{F}(\mathbf{x})\right]-\frac{1}{N\left(N^{2}-1\right)} \operatorname{Tr}\left[W^{F}(\mathbf{x}) W^{F \dagger}(\mathbf{y})\right]-1\right) \\
& \left.\left.\times\left(\frac{1}{N^{2}-1} \operatorname{Tr}\left[W^{F^{\dagger}}(\mathbf{x}) W^{F}(\mathbf{z})\right] \operatorname{Tr}\left[W^{F^{\dagger}}(\mathbf{z}) W^{F}(\mathbf{y})\right]-\frac{1}{N\left(N^{2}-1\right)} \operatorname{Tr}\left[W^{F}(\mathbf{y}) W^{F \dagger}(\mathbf{x})\right]-1\right)\right\rangle\right)
\end{aligned}
$$

This is the operatorial form of the low $x$ evolution equation for the diffractive photoabsorption cross section.

A comment is in order about the precise meaning of $P_{\text {diff }}$. We have defined $P_{\text {diff }}$ at $x_{0}$ as the probability that the $q \bar{q}$ pair in the photon wave function remains in a color singlet state after the interaction with the target. At lower value of $x$ the wave function contains a quark-antiquark-gluon component. The rapidity of this Weizsäcker-Williams gluon in the leading logarithmic approximation is always smaller than the rapidity of the quark or antiquark but greater than the initial rapidity $\ln \left(1 / x_{0}\right)$. Again we require that the outgoing state be in the color singlet. This requirement does not constrain the emission into the rapidity interval between the gluon and the quark-antiquark pair in the final state, but it does not allow emission of particles with rapidity smaller than that of the softest gluon, that is $\ln \left(1 / x_{0}\right)$. Thus the final states allowed by our definition have rapidity gap on the target side of at least $\ln \left(1 / x_{0}\right)$.

Our definition of $P_{\text {diff }}$ coincides therefore with that of Ref. [21] and Eq. (5.5) is the operator form of the evolution equation for this quantity. It is also easy to see that in the leading order in $1 / N$ this equation indeed coincides with the equation derived in Ref. [21]. The straightforward way is again to realize that in the leading order in $1 / N$ the target field averages factorize. Thus the right hand side of Eq. (5.5) can be factorized into products of

$$
P_{\text {diff }}=\left\langle\left\langle\left(\frac{1}{N} \operatorname{Tr}\left[W^{F}(\mathbf{x}) W^{F \dagger}(\mathbf{y})\right]-1\right)(\text { H.c. })\right\rangle\right\rangle
$$

(denoted in [21] by $N_{D}$ ) and

$$
P_{\text {tot }}=\left\langle\left\langle\left(1-\frac{1}{N} \operatorname{Tr}\left[W^{F}(\mathbf{x}) W^{F^{\dagger}}(\mathbf{y})\right]\right)\right\rangle\right\rangle
$$

(denoted in [21] by $N_{0}$ ). Neglecting the explicit $1 / N^{2}$ corrections in the coefficients of Eq. (5.5) the result is identical to the differential form of Eq. (11) of [21]:

$$
\begin{aligned}
\frac{d}{d \xi} P_{\mathrm{diff}}(x, y)= & C_{F} F^{2}\left\{-P_{\mathrm{diff}}(x, y)-P_{\mathrm{diff}}(x, z)-P_{\mathrm{diff}}(y, z)\right. \\
& +P_{\mathrm{diff}}(x, z) P_{\mathrm{diff}}(z, y)-2 P_{\mathrm{diff}}(x, z) P_{\mathrm{tot}}(z, y) \\
& \left.-2 P_{\mathrm{tot}}(x, z) P_{\mathrm{diff}}(z, y)+2 P_{\mathrm{tot}}(x, z) P_{\mathrm{tot}}(z, y)\right\} .
\end{aligned}
$$

We note however that within the region of validity of the present approximation, (5.6) is not really a differential equa-

\footnotetext{
${ }^{2}$ It has been recently suggested [26] that the apparent differences between the BK and JKLW equations are due to the incorrect derivation of the latter. However, since the discussion of [26] does not point to a mistake in the calculations of $[8,17]$, we consider the question open as discussed in [17].
} 
tion, but it rather determines $P_{\text {diff }}$ directly in terms of the dipole cross scattering probability $D$ of Eq. (5.2). The point is that the right hand side of Eq. (5.5) in the leading order in $1 / N$ can be written entirely in terms of $D$ :

$$
\begin{aligned}
\frac{d}{d \xi} P_{\text {diff }}(x, y)= & C_{F} F^{2}\left\{-D^{2}(x, y)+[D(x, z) D(z, y)\right. \\
& \left.-D(x, z)-D(z, y)]^{2}\right\} .
\end{aligned}
$$

Thus once $D(\xi)$ is found by solving Eq. (5.3), the diffractive probability can be found by directly integrating the right hand side of Eq. (5.7). The reason Eq. (5.7) is consistent with Eq. (5.6) is the following. The initial condition satisfied by $P_{\text {diff }}$ as discussed in [21] is

$$
P_{\text {diff }}\left(x=x_{0}\right)=D\left(x_{0}\right)^{2} .
$$

At lower values of $x$ the solution of Eq. (5.7) of course will not satisfy this relation any longer. However the difference between $P_{\text {diff }}$ and $D^{2}$ at any value of $x$ is small, of order $\ln \left(x_{0} / x\right) F^{2}$. Thus in the right hand side of the differential equation (5.6) the two can be interchanged as long as $\ln \left(x_{0} / x\right) \ll 1 / F^{2}$. As discussed in the previous subsection, this condition has to be satisfied anyway for the whole scheme to be consistent. For even lower values of $x$ the corrections nonlinear in $F^{2}$ (or "Pomeron loop" diagrams) become important, and those are not accounted for in the present approximation (as well as in the derivations of Refs. [7,21]).

Thus we stress again that in order to find the diffractive probability one does not have to solve an independent evolution equation, but rather integrate Eq. (5.7) with the known right hand side. This statement remains true beyond the leading order in $1 / N$. The complete set of $1 / N$ corrections due to correlated dipole propagation is read off Eq. (4.15):

$$
\begin{aligned}
\frac{d}{d \xi} P_{\text {diff }}(x, y)= & C_{F} F^{2}\left[-\frac{N^{2}-2}{N^{2}\left(N^{2}-1\right)}+2 e^{-C_{F} v(\mathbf{x}-\mathbf{y})}-\frac{\left(N^{2}-1\right)^{3}-1}{N^{2}\left(N^{2}-1\right)^{2}} e^{-N v(\mathbf{x}-\mathbf{y})}+\frac{N^{2}-2}{N^{2}\left(N^{2}-1\right)}\left(e^{-N v(\mathbf{z}-\mathbf{x})}+e^{-N v(\mathbf{z}-\mathbf{y})}\right)\right. \\
& +\frac{N^{2}-4}{2\left(N^{2}-1\right)} e^{-N[v(\mathbf{z}-\mathbf{x})+v(\mathbf{z}-\mathbf{y})]}-2 e^{(-N / 2)[v(\mathbf{z}-\mathbf{x})+v(\mathbf{z}-\mathbf{y})]}\left(e^{(1 / 2 N) v(\mathbf{x}-\mathbf{y})}-\frac{2}{N^{2}\left(N^{2}-1\right)} e^{(-N / 2) v(\mathbf{x}-\mathbf{y})}\right) \\
& \left.+\frac{N^{2}}{4\left(N^{2}-1\right)}\left(\frac{N+3}{N+1} e^{-(N+1)[v(\mathbf{z}-\mathbf{x})+v(\mathbf{z}-\mathbf{y})]+v(\mathbf{x}-\mathbf{y})}+\frac{N-3}{N-1} e^{-(N-1)[v(\mathbf{z}-\mathbf{x})+v(\mathbf{z}-\mathbf{y})]-v(\mathbf{x}-\mathbf{y})}\right)\right] .
\end{aligned}
$$

Here, $v(\mathbf{x}-\mathbf{y})$ is related to the total dipole cross section $D(\mathbf{x}-\mathbf{y})$ via Eq. (5.2).

\section{SUMMARY}

In this paper we have discussed in a very explicit and simple way the physics of high energy processes in the eikonal approximation. This provides a simple unifying framework for a variety of calculations existing in the literature. In particular we considered processes where a small projectile (a hadron or a virtual photon) scatters on a large target (a nucleus). Our approach then allowed us to derive the inclusive one particle spectrum as well as total, inelastic and diffractive cross sections. In particular the low $x$ evolution equation for the DIS cross section [7] follows immediately from this calculation. The various sources of the breakdown of this evolution equation at very low $x$ are also seen clearly in our derivation. We have also derived the operatorial form for the evolution of the diffractive cross section and have shown that in the large $1 / N$ limit it reduces to the equation of Ref. [21]. We have clarified that this evolution is not independent of the total photoabsorption cross section, and indeed the diffractive cross section is determined by a direct integration once the total dipole scattering probability (at fixed impact parameter) is known.
The averaging over the target nucleus wave function has been performed in the approximation where the target is considered to be a dilute system of weak sources of the gluon field. We did however calculate explicitly all-order $1 / N$ corrections in this model. This allowed us to provide all-order corrections to the various cross sections as well as to both evolution equations. These corrections stem from the fact that fundamental dipoles do not propagate through the target independently from each other. These corrections have not appeared in the literature so far.

We hope that this work is useful in providing a simple explicit unifying framework for the eikonal physics. The method discussed here allows simple calculations of many other observables at high energy. What we consider to be most interesting are less inclusive observables giving detailed information about the structure of the final states. It is for example straightforward to determine evolution of such quantities as the inclusive one particle distributions and cross sections for diffractive vector meson production [19].

\section{ACKNOWLEDGMENT}

We thank Yuri Kovchegov and Sangyong Jeon for interesting discussions and for pointing out typos in an earlier version of this manuscript. 


\section{APPENDIX A: PHOTOABSORPTION CROSS SECTIONS}

In this appendix, we give full expressions for the differential inelastic $(c=$ inel $)$, diffractive $(c=$ diff $)$ and total $(c=$ tot $)$ photoabsorption cross sections. We consider contributions from the $q \bar{q}$ and $q \bar{q} g$ Fock components of the virtual photon. We also give expressions for the differential cross sections with respect to the transverse momenta $\mathbf{p}_{1}, \mathbf{p}_{2}$, and $\mathbf{k}$ of the quark, antiquark and gluon respectively. The calculation of the medium average $\langle\langle\cdots\rangle\rangle$ will be discussed in Appendix $\mathrm{C}$.

The wave functions $\delta \Psi_{c}$ are constructed as discussed in Sec. II.

The $S$-matrix element or overlap (2.6) at fixed transverse coordinates reads

$$
\begin{aligned}
& \left\langle\Psi_{\text {in }}^{(q \bar{q}+q \bar{q} g)}(\mathbf{x}, \mathbf{y}) \mid \Psi_{\text {out }}^{(q \bar{q}+q \bar{q} g)}(\mathbf{x}, \mathbf{y})\right\rangle \\
& \quad=\left\langle\left\langle\frac{\mathcal{N}^{2}}{N} \operatorname{Tr}\left[W^{F^{\dagger}}(\mathbf{y}) W^{F}(\mathbf{x})\right]+\frac{1}{N} \ln \left(x_{0} / x\right) \int d^{2} \mathbf{z}|f(\mathbf{z}-\mathbf{x})-f(\mathbf{z}-\mathbf{y})|{ }^{2} \operatorname{Tr}\left[T^{a} W^{F}(\mathbf{x}) T^{b} W^{F \dagger}(\mathbf{y})\right] W_{a b}^{A}(\mathbf{z})\right\rangle\right\rangle .
\end{aligned}
$$

Treating higher powers of the WW fields $f$ in the normalization factor $\mathcal{N}$ as perturbatively small contributions which can be neglected, and using Eq. (B5), one finds

$$
\begin{aligned}
\left\langle\Psi_{\text {in }}^{(q \bar{q}+q \bar{q} g)}(\mathbf{x}, \mathbf{y}) \mid \Psi_{\text {out }}^{(q \bar{q}+q \bar{q} g)}(\mathbf{x}, \mathbf{y})\right\rangle= & \left\langle\left\langle\frac{1}{N} \operatorname{Tr}\left[W^{F \dagger}(\mathbf{y}) W^{F}(\mathbf{x})\right]+\frac{1}{2 N} \ln \left(x_{0} / x\right) \int d^{2} \mathbf{z}|f(\mathbf{z}-\mathbf{x})-f(\mathbf{z}-\mathbf{y})| 2\right.\right. \\
& \left.\left.\times\left\{\operatorname{Tr}\left[W^{F \dagger}(\mathbf{z}) W^{F}(\mathbf{x})\right] \operatorname{Tr}\left[W^{F^{\dagger}}(\mathbf{y}) W^{F}(\mathbf{z})\right]-N \operatorname{Tr}\left[W^{F \dagger}(\mathbf{y}) W^{F}(\mathbf{x})\right]\right\}\right\rangle\right) .
\end{aligned}
$$

For the diffractive cross section (2.17), we use the projection operator on the color singlet states

$$
\begin{aligned}
& \mathcal{P}_{\text {singlet }}^{q \bar{q}}=\frac{1}{N} \delta_{\alpha \bar{\alpha}}|\alpha, \bar{\alpha}\rangle\langle\beta, \bar{\beta}| \delta_{\beta \bar{\beta}}, \\
& \mathcal{P}_{\text {singlet }}^{q \bar{q} g}=\frac{2}{N^{2}-1} T_{\alpha \beta}^{a}|\alpha \beta a\rangle\langle\bar{\alpha} \bar{\beta} \bar{a}| T_{\bar{\alpha} \bar{\beta}}^{\bar{a}} .
\end{aligned}
$$

We start with the contribution from the $q \bar{q}$ component. The contribution to the total cross section reads

$$
\left\langle\delta \Psi_{\text {tot }}^{q \bar{q}}(\mathbf{x}, \mathbf{y}) \mid \delta \Psi_{\text {tot }}^{q \bar{q}}(\mathbf{x}, \mathbf{y})\right\rangle=\left\langle\left\langle 2-\frac{1}{N} \operatorname{Tr}\left[W^{F \dagger}(\mathbf{x}) W^{F}(\mathbf{y})\right]-\frac{1}{N} \operatorname{Tr}\left[W^{F \dagger}(\mathbf{y}) W^{F}(\mathbf{x})\right]\right\rangle\right\rangle
$$

For the inelastic cross section we have

$$
\left\langle\delta \Psi_{\text {inel }}^{q \bar{q}}(\mathbf{x}, \mathbf{y}) \mid \delta \Psi_{\text {inel }}^{q \bar{q}}(\mathbf{x}, \mathbf{y})\right\rangle=1-\left\langle\left\langle\frac{1}{N} \operatorname{Tr}\left[W^{F^{\dagger}}(\mathbf{x}) W^{F}(\mathbf{y})\right]\right\rangle\right\rangle\left\langle\left\langle\frac{1}{N} \operatorname{Tr}\left[W^{F^{\dagger}}(\mathbf{y}) W^{F}(\mathbf{x})\right]\right\rangle\right\rangle,
$$

while the projectile biased inelastic probability is

$$
\left\langle\delta \Psi_{\text {inel }}^{q \bar{q} p r o j}(\mathbf{x}, \mathbf{y}) \mid \delta \Psi_{\text {inel }}^{q \bar{q} p r o j}(\mathbf{x}, \mathbf{y})\right\rangle=1-\left\langle\left\langle\frac{1}{N^{2}} \operatorname{Tr}\left[W^{F \dagger}(\mathbf{x}) W^{F}(\mathbf{y})\right] \operatorname{Tr}\left[W^{F \dagger}(\mathbf{y}) W^{F}(\mathbf{x})\right]\right\rangle\right\rangle .
$$

Finally the diffractive probability is

$$
\left\langle\delta \Psi_{\text {diff }}^{q \bar{q}}(\mathbf{x}, \mathbf{y}) \mid \delta \Psi_{\text {diff }}^{q \bar{q}}(\mathbf{x}, \mathbf{y})\right\rangle=\left\langle\left\langle\left(\frac{1}{N} \operatorname{Tr}\left[W^{F}(\mathbf{x}) W^{F^{\dagger}}(\mathbf{y})\right]-1\right)\left(\frac{1}{N} \operatorname{Tr}\left[W^{F}(\mathbf{y}) W^{F \dagger}(\mathbf{x})\right]-1\right)\right\rangle\right\rangle .
$$

For the same expressions including the $q \bar{q} g$ component, we find 


$$
\begin{aligned}
& \left\langle\delta \Psi_{\text {tot }}^{(q \bar{q}+q \bar{q} g)}(\mathbf{x}, \mathbf{y}) \mid \delta \Psi_{\text {tot }}^{(q \bar{q}+q \bar{q} g)}(\mathbf{x}, \mathbf{y})\right\rangle=\left\langle\left\langle 2-\frac{1}{N} \operatorname{Tr}\left[W^{F^{\dagger}}(\mathbf{x}) W^{F}(\mathbf{y})\right]-\frac{1}{N} \operatorname{Tr}\left[W^{F \dagger}(\mathbf{y}) W^{F}(\mathbf{x})\right]\right.\right. \\
& -\frac{1}{2 N} \ln \left(x_{0} / x\right) F^{2}\left\{\operatorname{Tr}\left[W^{F^{\dagger}}(\mathbf{z}) W^{F}(\mathbf{x})\right] \operatorname{Tr}\left[W^{F^{\dagger}}(\mathbf{y}) W^{F}(\mathbf{z})\right]-N \operatorname{Tr}\left[W^{F^{\dagger}}(\mathbf{y}) W^{F}(\mathbf{x})\right]\right. \\
& \left.\left.\left.+\operatorname{Tr}\left[W^{F^{\dagger}}(\mathbf{x}) W^{F}(\mathbf{z})\right] \operatorname{Tr}\left[W^{F \dagger}(\mathbf{z}) W^{F}(\mathbf{y})\right]-N \operatorname{Tr}\left[W^{F^{\dagger}}(\mathbf{x}) W^{F}(\mathbf{y})\right]\right\}\right)\right\rangle, \\
& \left\langle\delta \Psi_{\text {inel }}^{(q \bar{q}+q \bar{q} g)}(\mathbf{x}, \mathbf{y}) \mid \delta \Psi_{\text {inel }}^{(q \bar{q}+q \bar{q} g)}(\mathbf{x}, \mathbf{y})\right\rangle=1-\frac{1}{N^{2}}\left\langle\left\langle\operatorname{Tr}\left[W^{F \dagger}(\mathbf{x}) W^{F}(\mathbf{y})\right]\right\rangle\right\rangle\left\langle\left\langle\operatorname{Tr}\left[W^{F^{\dagger}}(\mathbf{y}) W^{F}(\mathbf{x})\right]\right\rangle\right\rangle+\ln \left(x_{0} / x\right) F^{2} \frac{1}{2 N^{2}} \\
& \times\left\langle\left\langle\operatorname{Tr}\left[W^{F^{\dagger}}(\mathbf{y}) W^{F}(\mathbf{x})\right]\right\rangle\right\rangle\left\langle\left\langle\left\{ N \operatorname{Tr}\left[W^{F \dagger}(\mathbf{x}) W^{F}(\mathbf{y})\right]-\operatorname{Tr}\left[W^{F \dagger}(\mathbf{x}) W^{F}(\mathbf{z})\right]\right.\right.\right. \\
& \left.\left.\left.\times \operatorname{Tr}\left[W^{F \dagger}(\mathbf{z}) W^{F}(\mathbf{y})\right]\right\}\right\rangle\right\rangle+\ln \left(x_{0} / x\right) F^{2} \frac{1}{2 N^{2}}\left\langle\left\langle\operatorname{Tr}\left[W^{F^{\dagger}}(\mathbf{x}) W^{F}(\mathbf{y})\right]\right\rangle\right\rangle \\
& \times\left\langle\left\langle\left\{N \operatorname{Tr}\left[W^{F^{\dagger}}(\mathbf{y}) W^{F}(\mathbf{x})\right]-\operatorname{Tr}\left[W^{F^{\dagger}}(\mathbf{z}) W^{F}(\mathbf{x})\right] \operatorname{Tr}\left[W^{F^{\dagger}}(\mathbf{y}) W^{F}(\mathbf{z})\right]\right\}\right\rangle\right\rangle, \\
& \left\langle\delta \Psi_{\text {diff }}^{(q \bar{q}+q \bar{q} g)}(\mathbf{x}, \mathbf{y}) \mid \delta \Psi_{\text {diff }}^{(q \bar{q}+q \bar{q} g)}(\mathbf{x}, \mathbf{y})\right\rangle=\left\langle\left\langle\left(\frac{1}{N} \operatorname{Tr}\left[W^{F}(\mathbf{x}) W^{F \dagger}(\mathbf{y})\right]-1\right)\left(\frac{1}{N} \operatorname{Tr}\left[W^{F}(\mathbf{y}) W^{F \dagger}(\mathbf{x})\right]-1\right)\right.\right. \\
& +C_{F} \ln \left(x_{0} / x\right) F^{2}\left\{-\left(\frac{1}{N} \operatorname{Tr}\left[W^{F}(\mathbf{x}) W^{F \dagger}(\mathbf{y})\right]-1\right)\left(\frac{1}{N} \operatorname{Tr}\left[W^{F}(\mathbf{y}) W^{F \dagger}(\mathbf{x})\right]-1\right)\right. \\
& +\left(\frac{1}{N^{2}-1} \operatorname{Tr}\left[W^{F^{\dagger}}(\mathbf{y}) W^{F}(\mathbf{z})\right] \operatorname{Tr}\left[W^{F^{\dagger}}(\mathbf{z}) W^{F}(\mathbf{x})\right]-\frac{1}{N\left(N^{2}-1\right)} \operatorname{Tr}\left[W^{F}(\mathbf{x}) W^{F^{\dagger}}(\mathbf{y})\right]-1\right) \\
& \times\left(\frac{1}{N^{2}-1} \operatorname{Tr}\left[W^{F^{\dagger}}(\mathbf{x}) W^{F}(\mathbf{z})\right] \operatorname{Tr}\left[W^{F \dagger}(\mathbf{z}) W^{F}(\mathbf{y})\right]\right. \\
& \left.\left.\left.\left.-\frac{1}{N\left(N^{2}-1\right)} \operatorname{Tr}\left[W^{F}(\mathbf{y}) W^{F \dagger}(\mathbf{x})\right]-1\right)\right\}\right\rangle\right\}
\end{aligned}
$$

The virtual photon wave function in the approximation (4.5) contains at most one quark, one antiquark and one gluon. Thus the various differential cross sections are obtained form the appropriate number of particles by integration over the impact parameter. For example,

$$
\begin{aligned}
\frac{d \sigma_{\mathrm{c}}^{q \bar{q}}}{d \mathbf{p}_{1} d \mathbf{p}_{2}} & \left.=\int d^{2} \mathbf{x} d^{2} \mathbf{y}\left\langle\delta \Psi_{\mathrm{c}}^{q \bar{q}}(\mathbf{x}, \mathbf{y})\right| a_{q}^{\dagger}\left(\mathbf{p}_{1}\right) a_{q}\left(\mathbf{p}_{1}\right) a_{\bar{q}}^{\dagger}\left(\mathbf{p}_{2}\right) a_{q}^{-} \mathbf{p}_{2}\right)\left|\delta \Psi_{\mathrm{c}}^{q \bar{q}}(\mathbf{x}, \mathbf{y})\right\rangle \\
& =\int d z d^{2} \mathbf{x} d^{2} \mathbf{y} d^{2} \overline{\mathbf{x}} d^{2} \overline{\mathbf{y}} e^{i \mathbf{p}_{1}(\mathbf{x}-\overline{\mathbf{x}})+i \mathbf{p}_{2}(\mathbf{y}-\overline{\mathbf{y}})} \psi(\overline{\mathbf{x}}-\overline{\mathbf{y}}, z) \psi^{*}(\mathbf{x}-\mathbf{y}, z) P_{c}(\overline{\mathbf{x}}, \overline{\mathbf{y}}, \mathbf{x}, \mathbf{y})
\end{aligned}
$$

and

$$
\begin{aligned}
\frac{d \sigma_{\mathrm{c}}^{q \bar{q} g}}{d \mathbf{p}_{1} d \mathbf{p}_{2} d \mathbf{k}}= & \int d \mathbf{x} d \mathbf{y} d \mathbf{z} d \overline{\mathbf{x}} d \overline{\mathbf{y}} d \overline{\mathbf{z}} e^{i \mathbf{p}_{1}(\mathbf{x}-\overline{\mathbf{x}})+i \mathbf{p}_{2}(\mathbf{y}-\overline{\mathbf{y}})+i \mathbf{k}(\mathbf{z}-\overline{\mathbf{z}})}\left\langle\delta \Psi_{\mathrm{c}}^{q \bar{q} g}(\mathbf{x}, \mathbf{y}, \mathbf{z})\right| a_{q}^{\dagger}\left(\mathbf{p}_{1}\right) \\
& \times a_{q}\left(\mathbf{p}_{1}\right) a_{q}^{\dagger}\left(\mathbf{p}_{2}\right) a_{q}^{-}\left(\mathbf{p}_{2}\right) a^{\dagger}(\mathbf{k}) a(\mathbf{k})\left|\delta \Psi_{\mathrm{c}}^{q \bar{q} g}(\mathbf{x}, \mathbf{y}, \mathbf{z})\right\rangle \\
= & \int d z d^{2} \mathbf{x} d^{2} \mathbf{y} d^{2} \mathbf{z} d^{2} \overline{\mathbf{x}} d^{2} \overline{\mathbf{y}} d^{2} \overline{\mathbf{z}} e^{i \mathbf{p}_{1}(\mathbf{x}-\overline{\mathbf{x}})+i \mathbf{p}_{2}(\mathbf{y}-\overline{\mathbf{y}})+i \mathbf{k}(\mathbf{z}-\overline{\mathbf{z}})} \psi(\overline{\mathbf{x}}-\overline{\mathbf{y}}, \overline{\mathbf{z}}, z) \psi^{*}(\mathbf{x}-\mathbf{y}, \mathbf{z}, z) P_{c}(\overline{\mathbf{x}}, \overline{\mathbf{y}}, \overline{\mathbf{z}}, \mathbf{x}, \mathbf{y}, \mathbf{z}),
\end{aligned}
$$


where $a_{q}, a_{q}^{-}$and $a$ are annihilation operators of the quark, antiquark and gluon respectively. The wave function $\psi(\mathbf{x}-\mathbf{y}, z)$ is defined in Eq. (4.1) and $\psi(\mathbf{x}-\mathbf{y}, \mathbf{z}, z)$ can be read off Eqs. (4.1) and (4.3). Here $P_{c}$ are amplitudes given in the following in terms of the target gluon field averages. We give the expressions for the quark-antiquark-gluon probability $P_{c}(\overline{\mathbf{x}}, \overline{\mathbf{y}}, \overline{\mathbf{z}}, \mathbf{x}, \mathbf{y}, \mathbf{z})$, from which one can obtain the quark-gluon probability as

$$
P_{c}(\overline{\mathbf{x}}, \overline{\mathbf{y}}, \mathbf{x}, \mathbf{y})=\int d^{2} \mathbf{z} P_{c}(\overline{\mathbf{x}}, \overline{\mathbf{y}}, \mathbf{z}, \mathbf{x}, \mathbf{y}, \mathbf{z}) .
$$

We find

$$
\begin{aligned}
& P_{\text {tot }}(\overline{\mathbf{x}}, \overline{\mathbf{y}}, \overline{\mathbf{z}}, \mathbf{x}, \mathbf{y}, \mathbf{z})=\left\langle\left\langle\mathcal{N} \overline{\mathcal{N}}\left(\frac{1}{N} \operatorname{Tr}\left[W^{F \dagger}(\overline{\mathbf{x}}) W^{F}(\overline{\mathbf{y}}) W^{F \dagger}(\mathbf{y}) W^{F}(\mathbf{x})\right]-\frac{1}{N} \operatorname{Tr}\left[W^{F \dagger}(\overline{\mathbf{x}}) W^{F}(\overline{\mathbf{y}})\right]-\frac{1}{N} \operatorname{Tr}\left[W^{F^{\dagger}}(\mathbf{y}) W^{F}(\mathbf{x})\right]+1\right)\right.\right. \\
& +\frac{1}{N} F \bar{F}\left[W_{a b}^{A}(\mathbf{z}) W_{\bar{a} b}^{A}(\overline{\mathbf{z}}) \operatorname{Tr}\left[W^{F^{\dagger}}(\overline{\mathbf{x}}) T^{\bar{a}} W^{F}(\overline{\mathbf{y}}) W^{F^{\dagger}}(\mathbf{y}) T^{a} W^{F}(\mathbf{x})\right]-W_{a b}^{A}(\mathbf{z}) \operatorname{Tr}\left[T^{a} W^{F}(\mathbf{x}) T^{b} W^{F^{\dagger}}(\mathbf{y})\right]\right. \\
& \left.\left.-W_{a b}^{A}(\overline{\mathbf{z}}) \operatorname{Tr}\left[T^{a} W^{F}(\overline{\mathbf{y}}) T^{b} W^{F^{\dagger}}(\overline{\mathbf{x}})\right]+\frac{N^{2}-1}{2}\right]\right\rangle, \\
& P_{\text {inel }}(\overline{\mathbf{x}}, \overline{\mathbf{y}}, \overline{\mathbf{z}}, \mathbf{x}, \mathbf{y}, \mathbf{z})=\left\langle\left\langle\frac{\mathcal{N} \overline{\mathcal{N}}}{N} \operatorname{Tr}\left[W^{F^{\dagger}}(\overline{\mathbf{x}}) W^{F}(\overline{\mathbf{y}}) W^{F^{\dagger}}(\mathbf{y}) W^{F}(\mathbf{x})\right]-\left(1+\frac{C_{F}}{2}(F-\bar{F})^{2}\right) \frac{\mathcal{N}^{2} \overline{\mathcal{N}}^{2}}{N} \operatorname{Tr}\left[W^{F \dagger}(\overline{\mathbf{x}}) W^{F}(\overline{\mathbf{y}})\right]\right.\right. \\
& \times \frac{1}{N} \operatorname{Tr}\left[W^{F \dagger}(\mathbf{y}) W^{F}(\mathbf{x})\right]+\frac{1}{N} \bar{F} F \operatorname{Tr}\left[W^{F^{\dagger}}(\overline{\mathbf{x}}) T^{a} W^{F}(\overline{\mathbf{y}}) W^{F^{\dagger}}(\mathbf{y}) T^{b} W^{F}(\mathbf{x})\right] W_{a c}^{A}(\overline{\mathbf{z}}) W_{b c}^{A}(\mathbf{z}) \\
& -\frac{1}{N^{2}} F^{2} \operatorname{Tr}\left[W^{F^{\dagger}}(\overline{\mathbf{x}}) W^{F}(\overline{\mathbf{y}})\right] \operatorname{Tr}\left[T^{a} W^{F}(\mathbf{x}) T^{b} W^{F^{\dagger}}(\mathbf{y})\right] W_{a b}^{A}(\mathbf{z}) \\
& \left.\left.-\frac{1}{N^{2}} \bar{F}^{2} \operatorname{Tr}\left[W^{F}(\overline{\mathbf{y}}) T^{b} W^{F^{\dagger}}(\overline{\mathbf{x}}) T^{a}\right] W_{a b}^{A}(\overline{\mathbf{z}}) \operatorname{Tr}\left[W^{F^{\dagger}}(\mathbf{y}) W^{F}(\mathbf{x})\right]\right\rangle\right), \\
& P_{\text {diff }}(\overline{\mathbf{x}}, \overline{\mathbf{y}}, \mathbf{z}, \mathbf{x}, \mathbf{y}, \mathbf{z})=\left\langle\left\langle\mathcal{N} \overline{\mathcal{N}}\left(\frac{1}{N} \operatorname{Tr}\left[W^{F}(\mathbf{x}) W^{F^{\dagger}}(\mathbf{y})\right]-1\right)\left(\frac{1}{N} \operatorname{Tr}\left[W^{F}(\overline{\mathbf{y}}) W^{F^{\dagger}}(\overline{\mathbf{x}})\right]-1\right)\right.\right. \\
& +C_{F} F \bar{F}\left(\frac{2}{N^{2}-1} \operatorname{Tr}\left[W^{F \dagger}(\mathbf{y}) T^{a} W^{F}(\mathbf{x}) T^{b}\right] W_{a b}^{A}(\mathbf{z})-1\right) \\
& \left.\left.\times\left(\frac{2}{N^{2}-1} \operatorname{Tr}\left[W^{F \dagger}(\overline{\mathbf{x}}) T^{\bar{a}} W^{F}(\overline{\mathbf{y}}) T^{\bar{b}}\right] W_{\bar{a} \bar{b}}^{A}(\overline{\mathbf{z}})-1\right)\right)\right\rangle .
\end{aligned}
$$

Here we use the notation of Eqs. (4.4), (4.5) and $\bar{F}, \overline{\mathcal{N}}$ denote the analogous expressions at transverse coordinates $\overline{\mathbf{x}}, \overline{\mathbf{y}}$. All terms quadratic in $F$ (or $\bar{F}$ ) are understood to contain the factor $\ln \left(x_{0} / x\right)$. Also we have not distinguished between the true and the projectile biased inelastic amplitude, since it does not matter as long as we are interested in particles at rapidity far from the target.

\section{APPENDIX B: APPLICATION OF FIERZ IDENTITIES}

In order to simplify the color algebra in products of Wilson lines, we use the relation between adjoint and fundamental Wilson lines,

$$
W_{a b}^{A}(\mathbf{x})=2 \operatorname{Tr}\left[T^{a} W^{F}(\mathbf{x}) T^{b} W^{F \dagger}(\mathbf{x})\right],
$$

where the generators of the fundamental representation satisfy

$$
T^{a} T^{b}=\frac{1}{2 N} \delta_{a b}+\frac{1}{2} d_{a b c} T^{c}+i \frac{1}{2} f_{a b c} T^{c}
$$

We make extensive use of the Fierz identities

$$
T_{i j}^{a} T_{k l}^{a}=\frac{1}{2}\left(\delta_{i l} \delta_{j k}-\frac{1}{N} \delta_{i j} \delta_{k l}\right),
$$




$$
W_{i j}^{F}(\mathbf{x}) W_{k l}^{F \dagger}(\mathbf{x})=\frac{1}{N} \delta_{i l} \delta_{j k}+2 W_{a b}^{A}(\mathbf{x}) T_{i l}^{a} T_{k j}^{b}
$$

The fundamental Wilson lines in Eq. (B4) depend on the same transverse coordinate. This implies that transverse momentum integrations in cross sections of the type (A12) or (A13) are needed for this Fierz identity to apply. The simplifications which we shall obtain in using Eq. (B4) are technically identical to those obtained in a Feynman diagrammatic language via Mueller's technique of cancellation between real and virtual (contact) scattering contributions. Indeed, in Feynman diagrammatic language, the only prerequisite for application of Mueller's cancellations is again that parton lines be closed at the cut, i.e., that transverse coordinates of fundamental Wilson lines be equal in amplitude and complex conjugate amplitude [24]. The use of Eq. (B4) will allow us to circumvent explicit diagrammatic calculations.

Using the Fierz identities (B4), one can simplify, e.g., the following contributions to different DIS observables given in Appendix A:

$$
\begin{aligned}
& \operatorname{Tr}\left[W^{F}(\mathbf{z}) W^{F \dagger}(\mathbf{y})\right] \operatorname{Tr}\left[W^{F}(\mathbf{x}) W^{F \dagger}(\mathbf{z})\right] \\
& =\frac{1}{N} \operatorname{Tr}\left[W^{F}(\mathbf{x}) W^{F^{\dagger}}(\mathbf{y})\right]+2 W_{a b}^{A}(\mathbf{z}) \\
& \times \operatorname{Tr}\left[W^{F^{\dagger}}(\mathbf{y}) T^{a} W^{F}(\mathbf{x}) T^{b}\right], \\
& \operatorname{Tr}\left[W^{F}(\mathbf{x}) W^{F \dagger}(\mathbf{y})\right] \operatorname{Tr}\left[W^{F}(\mathbf{y}) W^{F \dagger}(\mathbf{x})\right] \\
& =1+W_{a b}^{A}(\mathbf{x}) W_{a b}^{A}(\mathbf{y}), \\
& \operatorname{Tr}\left[W^{F \dagger}(\mathbf{x}) W^{F}(\mathbf{y})\right] \operatorname{Tr}\left[T^{a} W^{F}(\mathbf{x}) T^{b} W^{F \dagger}(\mathbf{y})\right] W_{a b}^{A}(\mathbf{z}) \\
& =\frac{1}{2 N} W_{a b}^{A}(\mathbf{z}) W_{a b}^{A}(\mathbf{x})+\frac{1}{2 N} W_{a b}^{A}(\mathbf{z}) W_{a b}^{A}(\mathbf{y})+\frac{1}{4} W_{a b}^{A}(\mathbf{z}) \\
& \times W_{c d}^{A}(\mathbf{y}) W_{e f}^{A}(\mathbf{x})\left(d_{a c e}-i f_{a c e}\right)\left(d_{b d f}+i f_{b d f}\right),
\end{aligned}
$$

\section{APPENDIX C: TARGET AVERAGES}

To perform medium averages $\langle\langle\cdots\rangle\rangle$ over products of fundamental or adjoint Wilson lines, we expand the Wilson lines locally for each longitudinal position up to second order in the color potential. For the last position $\xi$ of the Wilson lines, one expands, e.g.,

$$
\begin{aligned}
W_{a b}^{A}(\mathbf{z}) & =V_{a b_{1}}^{A}(\mathbf{z})\left(\delta_{b_{1} b}-A^{g}(\xi, \mathbf{z}) f^{b_{1} g b}-\frac{C_{A}}{2} \delta_{b_{1} b} B(\xi, \mathbf{0})\right) \\
W^{F}(\mathbf{z}) & =V^{F}(\mathbf{z})\left(1+i A^{g}(\xi, \mathbf{z}) T^{g}-\frac{C_{F}}{2} B(\xi, \mathbf{0})\right) .
\end{aligned}
$$

Here, $V^{A}$ and $V^{F}$ denote the Wilson lines up to the last position $\xi$. Also, we have anticipated that in the medium averages color fields are expanded only up to second order locally in the longitudinal direction:

$$
\left\langle\left\langle A^{a}(\xi, \mathbf{z}) A^{b}\left(\xi, \mathbf{z}^{\prime}\right)\right\rangle\right\rangle=\delta^{a b} B\left(\xi, \mathbf{z}-\mathbf{z}^{\prime}\right) .
$$

In this way, one obtains, e.g., for the average of two fundamental or adjoint Wilson lines

$$
\begin{aligned}
\left\langle\left\langle\frac{1}{N} \operatorname{Tr}\left[W^{F \dagger}(\mathbf{x}) W^{F}(\mathbf{y})\right]\right\rangle\right\rangle \\
=\left\langle\left\langle\frac{1}{N} \operatorname{Tr}\left[V^{F \dagger}(\mathbf{x}) V^{F}(\mathbf{y})\right]\right\rangle\right\rangle \\
\quad \times\left\{1-C_{F}[B(\xi, \mathbf{0})-B(\xi, \mathbf{x}-\mathbf{y})]\right\} \\
=\exp \left[-C_{F} v(\mathbf{x}-\mathbf{y})\right], \\
\frac{1}{N^{2}-1}\left\langle\left\langle\operatorname{Tr}\left[W^{A \dagger}(\mathbf{y}) W^{A}(\mathbf{x})\right]\right\rangle\right\rangle_{t} \\
=\exp \left[-C_{A} v(\mathbf{x}-\mathbf{y})\right] .
\end{aligned}
$$

Here, the last line is obtained by reexponentiation with the help of 


$$
v(\mathbf{x})=\int d \xi[B(\xi, \mathbf{0})-B(\xi, \mathbf{x})]
$$

More complicated averages can be obtained in the same way:

$$
\begin{aligned}
\left\langle\left\langle W_{a b}^{A}(\mathbf{x}) \operatorname{Tr}\left[W^{F^{\dagger}}(\mathbf{y}) T^{a} W^{F}(\overline{\mathbf{y}}) T^{b}\right]\right\rangle\right\rangle & =\left\langle\left\langle V_{a b}^{A}(\mathbf{x}) \operatorname{Tr}\left[V^{F^{\dagger}}(\mathbf{y}) T^{a} V^{F}(\overline{\mathbf{y}}) T^{b}\right]\right\rangle\right\rangle \\
& \times\left[-\frac{C_{A}}{2}[B(\xi, \mathbf{0})+B(\xi, \mathbf{y}-\overline{\mathbf{y}})-B(\xi, \overline{\mathbf{y}}-\mathbf{x})\right. \\
& \left.-B(\xi, \mathbf{y}-\mathbf{x})]-C_{F}[B(\xi, \mathbf{0})-B(\xi, \mathbf{y}-\overline{\mathbf{y}})]\right] \\
= & \frac{N^{2}-1}{2} e^{\left(-C_{A} / 2\right)[v(\mathbf{x}-\overline{\mathbf{y}})+v(\mathbf{x}-\mathbf{y})]-\left(C_{F}-C_{A} / 2\right) v(\mathbf{y}-\overline{\mathbf{y}})} .
\end{aligned}
$$

As soon as color octets propagate along the products of Wilson lines, the calculations can become more involved. For the combinations of $f$ symbols

$$
\mathcal{F}_{b_{1} d_{1}}^{b d} \equiv f_{b_{1} n b} f_{d_{1} n d},
$$

which appear in the averaged Wilson lines, it is useful to observe that both singlet and octet structures are eigenstates of propagation:

$$
\begin{gathered}
\mathcal{F}_{b_{1} d_{1}}^{b d} \delta_{b d}=C_{A} \delta_{b_{1} d_{1}}, \\
\mathcal{F}_{b_{1} d_{1}}^{b d} f_{b d f}=\frac{C_{A}}{2} f_{b_{1} d_{1} f}, \\
\mathcal{F}_{b_{1} d_{1}}^{b d} d_{b d f}=\frac{C_{A}}{2} d_{b_{1} d_{1} f} .
\end{gathered}
$$

For an average of three Wilson lines, this leads to

$$
\begin{aligned}
\left(d_{a c e}-\right. & \left.i f_{a c e}\right)\left\langle\left\langle W_{a b}^{A}(\mathbf{z}) W_{c d}^{A}(\overline{\mathbf{z}}) W_{e f}^{A}(\mathbf{y})\right\rangle\right\rangle\left(d_{b d f}-i f_{b d f}\right) \\
= & \left(d_{a c e}-i f_{a c e}\right)\left\langle\left\langle V_{a b_{1}}^{A}(\mathbf{z}) V_{c d_{1}}^{A}(\overline{\mathbf{z}}) V_{e f_{1}}^{A}(\mathbf{y})\right\rangle\right\rangle\left[-\frac{3}{2} C_{A} B(\xi, \mathbf{0}) \delta_{b_{1} b} \delta_{d_{1} d} \delta_{f_{1} f}+B(\xi, \mathbf{z}-\overline{\mathbf{z}}) \mathcal{F}_{b_{1} d_{1}}^{b d} \delta_{f_{1} f}\right. \\
& \left.+B(\xi, \mathbf{z}-\mathbf{y}) \mathcal{F}_{b_{1} f_{1}}^{b f} \delta_{d_{1} d}+B(\xi, \overline{\mathbf{z}}-\mathbf{y}) \mathcal{F}_{d_{1} f_{1}}^{d f} \delta_{b_{1} b}\right]\left(d_{b d f}-i f_{b d f}\right) \\
= & \left(d_{a c e}-i f_{a c e}\right)\left\langle\left\langle V_{a b_{1}}^{A}(\mathbf{z}) V_{c d_{1}}^{A}(\overline{\mathbf{z}}) V_{e f_{1}}^{A}(\mathbf{y})\right\rangle\right\rangle\left(d_{b_{1} d_{1} f_{1}}-i f_{b_{1} d_{1} f_{1}}\right) \\
& -\left[\frac{3 C_{A}}{2} B(\xi, \mathbf{0})+\frac{C_{A}}{2} B(\xi, \mathbf{z}-\overline{\mathbf{z}})+\frac{C_{A}}{2} B(\xi, \mathbf{z}-\mathbf{y})+\frac{C_{A}}{2} B(\xi, \overline{\mathbf{z}}-\mathbf{y})\right] \\
= & \left(N^{2}-1\right)\left(2 N-\frac{4}{N}\right) \exp \left[-\frac{C_{A}}{2}[v(\mathbf{z}-\overline{\mathbf{z}})+v(\mathbf{z}-\mathbf{y})+v(\overline{\mathbf{z}}-\mathbf{y})] .\right.
\end{aligned}
$$

More complicated is the average over the four adjoint Wilson lines given in Eq. (B8). Again, we expand the Wilson lines according to Eq. (C1):

$$
\left\langle\left\langle W_{a b}^{A}(\mathbf{z}) W_{c d}^{A}(\mathbf{z}) W_{e f}^{A}(\mathbf{x}) W_{g h}^{A}(\mathbf{y})\right\rangle\right\rangle=\left\langle\left\langle V_{a \bar{a}}^{A}-(\mathbf{z}) V_{c c}^{A}-(\mathbf{z}) V_{e e^{-}}^{A}(\mathbf{x}) V_{g g}^{A}-(\mathbf{y})\right\rangle\right\rangle M_{b d f h}^{\overline{a c e} g} .
$$

We introduce the shorthand

$$
R_{a}=-v(\mathbf{z}-\mathbf{x})-v(\mathbf{z}-\mathbf{y}), \quad R_{b}=-v(\mathbf{x}-\mathbf{y}) .
$$

One eigenstate of $M_{b d f h}^{a c e g}$ is rather easily checked:

$$
M_{b d f h}^{a c e g}\left(f_{f b n} d_{h d n}+d_{f b n} f_{h d n}\right)=R_{a}\left(f_{e a n} d_{g c n}+d_{e a n} f_{g c n}\right) .
$$

To find the other eigenstates of $M_{b d f h}^{a c e g}$, we introduce the vectors

$$
\begin{aligned}
& u_{1}=\delta_{e a} \delta_{g c}, \quad u_{2}=\delta_{c a} \delta_{g e}, \quad u_{3}=\delta_{g a} \delta_{e c}, \\
& v_{1}=f_{e a n} f_{g c n}, \quad v_{2}=f_{c a n} f_{g e n}, \quad v_{3}=f_{g a n} f_{e c n}, \\
& w_{1}=d_{e a n} d_{g c n}, \quad w_{2}=d_{c a n} d_{g e n}, \quad w_{3}=d_{g a n} d_{e c n} .
\end{aligned}
$$

Color algebra implies the identities 


$$
\begin{aligned}
& v_{1}=\frac{2}{N}\left(u_{2}-u_{3}\right)+w_{2}-w_{3}, \\
& v_{2}=\frac{2}{N}\left(u_{1}-u_{3}\right)+w_{1}-w_{3}, \\
& v_{3}=\frac{2}{N}\left(u_{2}-u_{1}\right)+w_{2}-w_{1} .
\end{aligned}
$$

In the six-dimensional space, given by the vectors $\left(u_{1}, u_{2}, u_{3}, w_{1}, w_{2}, w_{3}\right)$, one finds by explicit calculation that the matrix $M_{b d f h}^{a c e g}$ acts like (using $R_{d}=R_{b}-R_{a}$ )

$$
\left(\begin{array}{cccccc}
N R_{a} & 0 & -\frac{2}{N} R_{d} & 0 & 0 & R_{d}\left(\frac{4}{N^{2}}-1\right) \\
\frac{2}{N} R_{d} & N R_{b} & \frac{2}{N} R_{d} & R_{d}\left(\frac{4}{N^{2}}-1\right) & 0 & R_{d}\left(\frac{4}{N^{2}}-1\right) \\
-\frac{2}{N} R_{d} & 0 & N R_{a} & R_{d}\left(\frac{4}{N^{2}}-1\right) & 0 & 0 \\
0 & 0 & R_{d} & \frac{N}{4}\left(3 R_{a}+R_{b}\right) & 0 & R_{d}\left(\frac{2}{N}-\frac{N}{4}\right) \\
R_{d} & 0 & R_{d} & -R_{d}\left(\frac{2}{N}-\frac{N}{4}\right) & \frac{N}{2}\left(R_{a}+R_{b}\right) & -R_{d}\left(\frac{2}{N}-\frac{N}{4}\right) \\
R_{d} & 0 & 0 & R_{d}\left(\frac{2}{N}-\frac{N}{4}\right) & 0 & \frac{N}{4}\left(3 R_{a}+R_{b}\right)
\end{array}\right)
$$

To calculate the average in Eq. (B8), we have to propagate the vector

$$
\left[\frac{2}{N} \delta_{f b} \delta_{h d}+d_{f b n} d_{h d n}-f_{f b n} f_{h d n}\right]=\frac{2}{N}\left(u_{1}-u_{2}+u_{3}\right)+w_{1}-w_{2}+w_{3} .
$$

Finding the eigenvectors and eigenvalues of Eq. (C22) with the help of MATHEMATICA, decomposing the vector (C23) in terms of the eigenvectors of Eq. (C22) and reexponentiating the obtained expressions, we find

$$
\begin{aligned}
& \frac{1}{16}\left\langle\left\langle W_{a b}^{A}(\mathbf{z}) W_{c d}^{A}(\mathbf{z}) W_{e f}^{A}(\mathbf{x}) W_{g h}^{A}(\mathbf{y})\right\rangle\right\rangle\left[\frac{2}{N} \delta_{a e} \delta_{c g}+\left(d_{a e m}+i f_{a e m}\right)\left(d_{c g m}+i f_{c g m}\right)\right]\left[\frac{2}{N} \delta_{f b} \delta_{h d}+\left(d_{f b n}+i f_{f b n}\right)\left(d_{h d n}+i f_{h d n}\right)\right] \\
& =\frac{1}{4 N^{2}} e^{N R_{b}}+\frac{(N-1)(N+3) N^{2}}{16} e^{N R_{a}+R_{a}-R_{b}}+\frac{N^{2}-1}{N^{2}} e^{N / 2\left(R_{a}+R_{b}\right)}+\frac{(N+1)(N-3) N^{2}}{16} e^{N R_{a}-R_{a}+R_{b}} \\
& +\frac{N\left(N^{2}-1\right)\left(N-\frac{4}{N}\right)}{8} e^{N R_{a}}
\end{aligned}
$$

This enters the calculation of the probability $P_{\text {diff }}^{(q \bar{q}+q \bar{q} g)}$ in Eq. (4.15) and the diffractive evolution equation (5.9).

Finally we present averaged expressions for the single differential quark cross sections, obtained from Eqs. (A15), (A17) by taking $\bar{y}=y$ :

$$
\frac{d \sigma_{\mathrm{tot}}^{q \bar{q}}}{d \mathbf{p}}=\int d \mathbf{x} d \mathbf{y} d \overline{\mathbf{x}} e^{i \mathbf{p}(\mathbf{x}-\overline{\mathbf{x}})}\left(1+e^{-C_{F} v(\overline{\mathbf{x}}-\mathbf{x})}-e^{-C_{F} v(\overline{\mathbf{x}}-\mathbf{y})}-e^{-C_{F} v(\mathbf{x}-\mathbf{y})}\right)
$$




$$
\begin{aligned}
\frac{d \sigma_{\mathrm{diff}}^{q \bar{q}}}{d \mathbf{p}}= & \int d \mathbf{x} d \mathbf{y} d \overline{\mathbf{y}} e^{i \mathbf{p}(\mathbf{y}-\overline{\mathbf{y}})}\left(\frac{1}{N^{2}} e^{-C_{F} v(\overline{\mathbf{y}}-\mathbf{y})}-e^{-C_{F} v(\overline{\mathbf{x}}-\mathbf{y})}-e^{-C_{F} v(\overline{\mathbf{x}}-\overline{\mathbf{y}})}\right. \\
& \left.+\frac{N^{2}-1}{N^{2}} e^{\left(-C_{A} / 2\right)[v(\mathbf{x}-\overline{\mathbf{y}})+v(\mathbf{x}-\mathbf{y})]-\left(C_{F}-C_{A} / 2\right) v(\mathbf{y}-\overline{\mathbf{y}})}+1\right) .
\end{aligned}
$$

[1] L. V. Gribov, E. M. Levin, and M. G. Ryskin, Phys. Rep. 100, 1 (1983); I. A. H. Mueller and Jian-wei Qiu, Nucl. Phys. B268, 427 (1986).

[2] R. Baier, Yu. L. Dokshitzer, A. H. Mueller, S. Peigne, and D. Schiff, Nucl. Phys. B483, 291 (1997); B484, 265 (1997); B531, 403 (1998); B. G. Zakharov, JETP Lett. 65, 615 (1997); Phys. At. Nucl. 61, 838 (1998) [Yad. Fiz. 61, 924 (1998)]; U. A. Wiedemann and M. Gyulassy, Nucl. Phys. B560, 345 (1999); U. A. Wiedemann, ibid. B588, 303 (2000); M. Gyulassy, P. Levai, and I. Vitev, ibid. B571, 197 (2000); ibid. B594, 371 (2001).

[3] W. Buchmüller and A. Hebecker, Nucl. Phys. B476, 203 (1996); W. Buchmüller, M. F. McDermott, and A. Hebecker, ibid. B487, 283 (1997); B500, 621(E) (1997); A. Hebecker, ibid. B505, 349 (1997); W. Buchmüller, T. Gehrmann, and A. Hebecker, ibid. B537, 477 (1999).

[4] L. McLerran and R. Venugopalan, Phys. Rev. D 49, 2233 (1994); 49, 3352 (1994); 50, 2225 (1999).

[5] Yu. V. Kovchegov and A. H. Mueller, Nucl. Phys. B529, 451 (1998).

[6] E. Gotsman, E. Levin, M. Lublinsky, U. Maor, E. Naftali, and K. Tuchin, hep-ph/0101344; E. Levin and K. Tuchin, hep-ph/0101275; A. L. Ayala, M. B. Gay Ducati, and E. M. Levin, Nucl. Phys. B493, 305 (1997); E. Gotsman, E. Levin, and U. Maor, Phys. Rev. D 60, 094011 (1999).

[7] I. Balitsky, Nucl. Phys. B463, 99 (1996); Phys. Rev. Lett. 81, 2024 (1998); Yu. V. Kovchegov, Phys. Rev. D 60, 034008 (1999); H. Weigert, hep-ph/0004044.

[8] J. Jalilian-Marian, A. Kovner, A. Leonidov, and H. Weigert, Nucl. Phys. B504, 415 (1997); Phys. Rev. D 59, 014014 (1999); 59, 034007 (1999); J. Jalilian-Marian, A. Kovner, and H. Weigert, ibid. 59, 014015 (1999); A. Kovner and J. G. Milhano, ibid. 61, 014012 (2000).

[9] A. Capella, E. G. Ferriero, C. A. Salgado, and A. B. Kaidalov,
Nucl. Phys. B593, 336 (2001); Phys. Rev. D 63, 054010 (2001).

[10] A. Makhlin and E. Surdutovich, Phys. Rev. C 58, 389 (1998); ibid.63, 044904 (2001); A. Makhlin, ibid. 63, 044902 (2001); ibid.63, 044903 (2001).

[11] L. Frankfurt and M. Strikman, Eur. Phys. J. A 5, 293 (1999); L. Frankfurt, V. Guzey, M. McDermott, and M. Strikman, hep-ph/0404252.

[12] A. Krasnitz and R. Venugopalan, Nucl. Phys. B557, 237 (1999); Phys. Rev. Lett. 84, 4309 (2000); 86, 1717 (2001).

[13] C. S. Lam and G. Mahlon, Phys. Rev. D 61, 014005 (2000); 62, 114023 (2000); 64, 016004 (2001).

[14] B. Z. Kopeliovich, J. Raufeisen, and A. V. Tarasov, Phys. Lett. B 503, 91 (2001); B. Z. Kopeliovich, A. V. Tarasov, and J. Hüfner, hep-ph/0104256.

[15] H. Navelet and R. Peschanski, hep-ph/0105030.

[16] Yu. V. Kovchegov, hep-ph/0011252.

[17] A. Kovner, J. G. Milhano, and H. Weigert, Phys. Rev. D 62, 114005 (2000).

[18] V. N. Gribov, Sov. Phys. JETP 29, 483 (1969).

[19] A. Kovner and U. A. Wiedemann (in progress).

[20] Yu. V. Kovchegov and L. McLerran, Phys. Rev. D 60, 054025 (1999); 62, 019901(E) (2000).

[21] Yu. V. Kovchegov and E. Levin, Nucl. Phys. B577, 221 (2000).

[22] J. Jalilian-Marian, A. Kovner, L. McLerran, and H. Weigert, Phys. Rev. D 55, 5414 (1997); Yu. V. Kovchegov, ibid. 54, 5463 (1996).

[23] J. F. Gunion and G. Bertsch, Phys. Rev. D 25, 746 (1982).

[24] U. A. Wiedemann, Nucl. Phys. B582, 409 (2000).

[25] L. Motyka, talk at "Beyond the Pomeron" workshop, Brookhaven, 2001.

[26] E. Iancu, A. Leonidov, and L. McLerran, hep-ph/0011241; Phys. Lett. B 510, 133 (2001). 